\title{
Article \\ Identification of Survival-Specific Genes in Clear Cell Renal Cell Carcinoma Using a Customized Next-Generation Sequencing Gene Panel
}

\author{
Jia Hwang ${ }^{1}$, Heeeun Kim ${ }^{1}$, Jinseon Han ${ }^{1}$, Jieun Lee ${ }^{1}$, Sunghoo Hong ${ }^{2}$, Saewoong Kim ${ }^{2}$, Sungjoo Kim Yoon ${ }^{3}$,

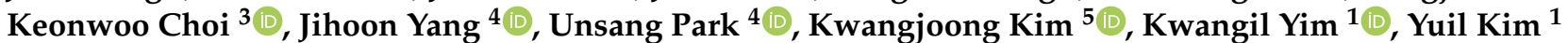 \\ and Yeongjin Choi ${ }^{1, * \mathbb{D}}$ \\ 1 Department of Hospital Pathology, Seoul St. Mary's Hospital, College of Medicine, \\ The Catholic University of Korea, Seoul 06591, Korea; hja0329@hotmail.com (J.H.); \\ heya0808@gmail.com (H.K.); jinseon-han@hotmail.com (J.H.); jning@naver.com (J.L.); \\ kangse_manse@catholic.ac.kr (K.Y.); yuil.gim@gmail.com (Y.K.) \\ 2 Department of Urology, Seoul St. Mary's Hospital, College of Medicine, The Catholic University of Korea, \\ Seoul 06591, Korea; toomey@catholic.ac.kr (S.H.); ksw1227@catholic.ac.kr (S.K.) \\ 3 Department of Biomedicine and Health Sciences, College of Medicine, The Catholic University of Korea, \\ Seoul 06591, Korea; sjkyoon@catholic.ac.kr (S.K.Y.); icsoo123@naver.com (K.C.) \\ 4 Department of Computer Science and Engineering, Sogang University, Seoul 04107, Korea; \\ yangjh@sogang.ac.kr (J.Y.); unsangpark@sogang.ac.kr (U.P.) \\ $5 \quad$ NGeneBio Co., Ltd., Seoul 08390, Korea; kjoong.kim@ngenebio.com \\ * Correspondence: mdyjchoi@catholic.ac.kr; Tel.: +82-(22)-2581616; Fax: +82-(22)-2581627
}

Citation: Hwang, J.; Kim, H.; Han, J.; Lee, J.; Hong, S.; Kim, S.; Yoon, S.K.;

Choi, K.; Yang, J.; Park, U.; et al.

Identification of Survival-Specific Genes in Clear Cell Renal Cell Carcinoma Using a Customized Next-Generation Sequencing Gene Panel. J. Pers. Med. 2022, 12, 113. https://doi.org/10.3390/ jpm12010113

Academic Editors: José I. López and Claudia Manini

Received: 16 November 2021

Accepted: 10 January 2022

Published: 14 January 2022

Publisher's Note: MDPI stays neutral with regard to jurisdictional claims in published maps and institutional affiliations.

Copyright: (c) 2022 by the authors. Licensee MDPI, Basel, Switzerland. This article is an open access article distributed under the terms and conditions of the Creative Commons Attribution (CC BY) license (https:// creativecommons.org/licenses/by/ $4.0 /)$.

\begin{abstract}
Purpose: Although mutations are associated with carcinogenesis, little is known about survival-specific genes in clear cell renal cell carcinoma (ccRCC). We developed a customized nextgeneration sequencing (NGS) gene panel with 156 genes. The purpose of this study was to investigate whether the survival-specific genes we found were present in Korean ccRCC patients, and their association with clinicopathological findings. Materials and Methods: DNA was extracted from the formalin-fixed, paraffin-embedded tissue of 22 ccRCC patients. NGS was performed using our survival-specific gene panel with an Illumina MiSeq. We analyzed NGS data and the correlations between mutations and clinicopathological findings and also compared them with data from the Cancer Genome Atlas-Kidney Renal Clear Cell Carcinoma (TCGA-KIRC) and Renal Cell CancerEuropean Union (RECA-EU). Results: We found a total of 100 mutations in 37 of the 156 genes $(23.7 \%)$ in 22 ccRCC patients. Of the 37 mutated genes, 11 were identified as clinicopathologically significant. Six were novel survival-specific genes (ADAMTS10, CARD6, NLRP2, OBSCN, SECISBP2L, and USP40), and five were top-ranked mutated genes (AKAP9, ARID1A, BAP1, KDM5C, and SETD2). Only CARD6 was validated as an overall survival-specific gene in this Korean study $(p=0.04$, $r=-0.441)$, TCGA-KIRC cohort $(p=0.0003)$, RECA-EU $(p=0.0005)$. The 10 remaining gene mutations were associated with clinicopathological findings; disease-free survival, mortality, nuclear grade, sarcomatoid component, N-stage, sex, and tumor size. Conclusions: We discovered 11 survivalspecific genes in cCRCC using data from TCGA-KIRC, RECA-EU, and Korean patients. We are the first to find a correlation between CARD6 and overall survival in ccRCC. The 11 genes, including $C A R D 6, N L R P 2, O B S C N$, and USP40, could be useful diagnostic, prognostic, and therapeutic markers in $\mathrm{CCRCC}$.
\end{abstract}

Keywords: clear cell renal cell carcinoma; survival; genes; mutations; CARD6; NGS; Korea

\section{Introduction}

Clear cell renal cell carcinoma (ccRCC) is one of the most lethal cancer types globally, accounting for 70-75\% of all renal cancers [1]. In South Korea, ccRCC accounted for approximately $85 \%$ of all renal cell carcinoma (RCC) cases in 2015 [2]. Several studies on 
ccRCC-related mutant genes have been reported based on next-generation sequencing (NGS) techniques, but most of them were performed on a small scale [3].

The clinical, morphological, and genetic characteristics of RCC have been found to vary significantly from patient to patient due to high tumor heterogeneity [4]. It has been widely known that cancer is caused by the accumulation of cancer-causing gene mutations, and in fact, at least one protein-altering mutation is found in $85 \%$ of cancer tissues [5]. Therefore, a diverse clinical picture arises due to the diversity of genetic mutations.

Recently, various genetic mutations causing RCC have been identified by high-throughput sequencing methods [6-10], such as those used in The Cancer Genome Atlas Kidney Renal Clear Cell Carcinoma (TCGA-KIRC) and Renal Cell Cancer-EU/FR (RECA-EU) databases, and many predisposing genes involved in the development of RCC have been reported through hereditary renal cancer studies [6,8-11]. However, studies on the most important gene mutations related to the survival of RCC patients are lacking, and most genetic studies are based on databases from Western populations. Few mutation data are based on the East Asian population.

Currently, NGS, which analyzes multiple genes in parallel at the same time, is the most useful method for diagnosing gene mutations [12,13]. To date, little is known about genetic markers that predict survival in patients with ccRCC. However, the application of personalized therapy requires the development of dedicated NGS panels for key target genes associated with important clinicopathological conditions, including survival.

In this study, we developed a customized NGS gene panel for ccRCC using 156 genes. This panel included 123 survival-specific genes newly discovered by machine learning, and 21 of the top-ranked mutated genes above 5\% in TCGA-KIRC, as well as genes from other databases. We used this panel to determine whether survival-specific gene mutations are present in Korean ccRCC patients and to analyze their association with clinicopathological findings. We discovered and validated 11 survival-specific genes in ccRCC patients based on TCGA-KIRC and RECA-EU data as well as data from Korean patients. To our knowledge, we are the first group to report a correlation between CARD6 mutation and overall survival (OS) in patients with ccRCC. We believe that the 11 genes, especially CARD6, NLRP2, OBSCN, and USP40, could be used as useful diagnostic, prognostic, and therapeutic markers in ccRCC.

\section{Materials and Methods}

\subsection{Ethical Statement}

All procedures performed in this study were in accordance with the 1964 Helsinki declaration and its later amendments or comparable ethical standards, and with the ethical standards approved by the Institutional Review Board of Catholic University of Korea, Seoul St. Mary's Hospital (approval no. 2018-2550-0008, date of approval: 25 September 2020). The retrospective genetic study and the treatment plan were conducted according to clinical guidelines and standard of care. The present genetic study results did not affect the treatment plan of patients following surgery. Informed written consent was provided by all patients.

\subsection{Machine Learning and Statistical Methods for the Discovery of 123 Survival-Specific Genes}

We conducted machine learning (ML) using Rapidminer (7.3 version, Boston, MA, USA) to discover survival-specific mutations in ccRCC from the TCGA database. From TCGAKIRC, 417 patients with both somatic nonsilent mutations and clinical-related data were selected. The clinical data were obtained from the TCGA portal, and the Mutation Annotation Formatted file was obtained from UCSC Xena (UCSC, https: / xenabrowser.net/). UCSC Xena data provided clinical information on mutations in 39,532 genes of 417 patients. ML techniques of three feature selection methods (Information Gain, Chi-squared test, and Minimum Redundancy Maximum Relevance (MRMR)) and three classifiers (Naïve Bayes, K-Nearest Neighbor (K-NN), and Support Vector Machine (SVM)) were implemented. The performances of the used models, shown in Table S1 (a)-(c), were compared and analyzed using survival graphs of the top 100 mutant genes selected by each model (Figure S1). 
The best-performing ML models were repeatedly utilized, and a total of 195 mutant genes related to the six clinicopathologic factors (age, sex, stage, recurrence, metastasis, and survival) were selected. To increase the accuracy, only genes commonly found by the three methods were extracted. Of 195 genes, 123 statistically validated genes were selected by analysis of variance (ANOVA) and a Fisher's exact test after analyzing their mutation frequencies and overall survival (OS) and disease-free survival (DFS) (Table S2).

\subsection{Patients}

Patients were enrolled from Catholic University College of Medicine, Seoul, Republic of Korea. The inclusion criteria of the study were patients who received radical/partial nephrectomy and pathology diagnosis of ccRCC and were willing to participate and provide signed informed consent. All patients with a renal tumor diagnosis underwent radical nephrectomy according to clinical indications. The pathology of each tumor was reviewed by pathologists specializing in kidney cancer identification, and only those diagnosed as ccRCC were included in the study. We selected 22 patients with ccRCC for this study. Clinicopathological factors of sex, age, tumor size, nuclear grade, sarcomatoid component, TNM stage $(\mathrm{T}=$ extent of the primary tumor, $\mathrm{N}=$ regional lymph nodes, $\mathrm{M}=$ distant metastases), and response to laparoscopic radical nephrectomy (LRN), and their results, are summarized in Table 1. Clinical demographic parameters; cancer stage using the American Joint Committee on Cancer; and pathological data of tumor stage, lymph node status, and Fuhrman nuclear grade were collected.

Table 1. Clinicopathological findings of 22 Korean patients with clear cell renal cell carcinoma.

\begin{tabular}{|c|c|c|c|c|c|c|c|c|c|c|c|}
\hline & P01 & P02 & P03 & P04 & P05 & P06 & P07 & P08 & P09 & P10 & P11 \\
\hline Age & 68 & 60 & 65 & 72 & 69 & 33 & 42 & 40 & 52 & 57 & 68 \\
\hline $\operatorname{Sex}(\mathrm{F} / \mathrm{M})$ & $\mathrm{F}$ & $\mathrm{F}$ & $\mathrm{M}$ & $\mathrm{F}$ & $\mathrm{M}$ & $\mathrm{F}$ & $\mathrm{F}$ & M & $\mathrm{M}$ & $\mathrm{M}$ & $\mathrm{M}$ \\
\hline Tumor Size $(\mathrm{cm})$ & 2.5 & 2.5 & 3.9 & 11 & 5.5 & 4.3 & 6 & 5.3 & 8.8 & 5.2 & 10 \\
\hline Nuclear Grade & II & II & IV & IV & III & II & II & III & III & III & IV \\
\hline $\begin{array}{l}\text { Sarcomatoid } \\
\text { component }\end{array}$ & 0 & 0 & 0 & 0 & 1 & 0 & 0 & 0 & 0 & 0 & 0 \\
\hline TNM T-Stage & T1a & T1a & T3a & $\mathrm{T} 2 \mathrm{~b}$ & $\mathrm{~T} 1 \mathrm{~b}$ & $\mathrm{~T} 1 \mathrm{~b}$ & $\mathrm{~T} 1 \mathrm{~b}$ & $\mathrm{~T} 1 \mathrm{~b}$ & $\mathrm{~T} 2 \mathrm{a}$ & T3a & T3a \\
\hline TNM N-Stage & 0 & 0 & 0 & 0 & 0 & 0 & 0 & 0 & 0 & 0 & 1 \\
\hline TNM M-Stage & 0 & 0 & 0 & 0 & 1 & 0 & 0 & 0 & 1 & 0 & 1 \\
\hline Recurrence & No & No & No & No & $\begin{array}{l}\text { Lung, } \\
\text { Bone }\end{array}$ & No & No & No & $\begin{array}{l}\text { Liver, Brain, } \\
\text { Lung }\end{array}$ & No & $\begin{array}{c}\text { Lung, Liver, Abdomen, } \\
\text { Retroperitoneum }\end{array}$ \\
\hline $\begin{array}{l}\text { Overall Survival } \\
\text { (Month) }\end{array}$ & 36 & 35 & 35 & 35 & 32 & 32 & 30 & 28 & 12 & 14 & 42 \\
\hline $\begin{array}{c}\text { Disease Free } \\
\text { Survival (Month) }\end{array}$ & 36 & 35 & 35 & 35 & 10 & 32 & 30 & 28 & 10 & 14 & 10 \\
\hline Death & $\mathrm{N}$ & $\mathrm{N}$ & $\mathrm{N}$ & $\mathrm{N}$ & $\mathrm{N}$ & $\mathrm{N}$ & $\mathrm{N}$ & $\mathrm{N}$ & $\mathrm{Y}$ & $\mathrm{N}$ & $\mathrm{Y}$ \\
\hline \multirow[t]{2}{*}{ Response to LRN * } & NED & NED & NED & NED & Fail & NED & NED & NED & Fail & NED & Fail \\
\hline & P12 & P13 & P14 & P15 & P16 & P17 & P18 & P19 & P20 & P21 & P22 \\
\hline Age & 67 & 62 & 51 & 40 & 82 & 82 & 65 & 47 & 45 & 82 & 61 \\
\hline $\operatorname{Sex}(\mathrm{F} / \mathrm{M})$ & $\mathrm{F}$ & $\bar{M}$ & M & M & $\mathrm{F}$ & $\bar{M}$ & $\mathrm{M}$ & M & $\mathrm{M}$ & $\mathrm{M}$ & M \\
\hline Tumor Size (cm) & 6 & 10 & 5 & 4.2 & 5.5 & 4.7 & 14.5 & 10.3 & 7.5 & 7.2 & 5.4 \\
\hline Nuclear Grade & IV & III & III & III & II & III & II & III & III & III & IV \\
\hline $\begin{array}{l}\text { Sarcomatoid } \\
\text { component }\end{array}$ & 1 & 0 & 0 & 0 & 0 & 0 & 0 & 0 & 0 & 0 & 0 \\
\hline TNM T-Stage & $\mathrm{T} 1 \mathrm{~b}$ & $\mathrm{~T} 2 \mathrm{a}$ & T3a & T3a & Т3a & Т3a & $\mathrm{T} 3 \mathrm{~b}$ & Т3a & $\mathrm{T} 2 \mathrm{a}$ & $\mathrm{T} 2 \mathrm{a}$ & $\mathrm{T} 3 \mathrm{~b}$ \\
\hline TNM N-Stage & 0 & 0 & 0 & 0 & 0 & 0 & 0 & 0 & 0 & 0 & 0 \\
\hline TNM M-Stage & 1 & 1 & 1 & 0 & 1 & 0 & 0 & 1 & 0 & 1 & 1 \\
\hline Recurrence & $\begin{array}{l}\text { Lung, } \\
\text { Bone }\end{array}$ & Lung & $\begin{array}{l}\text { Lung, } \\
\text { Brain }\end{array}$ & No & Lung & No & No & Lung & No & Lung & $\begin{array}{l}\text { Lung, Bone, Jejunum, } \\
\text { Peritoneum }\end{array}$ \\
\hline $\begin{array}{l}\text { Overall Survival } \\
\text { (Month) }\end{array}$ & 42 & 38 & 37 & 36 & 14 & 38 & 48 & 50 & 45 & 48 & 34 \\
\hline $\begin{array}{c}\text { Disease Free } \\
\text { Survival (Month) }\end{array}$ & 0 & 1 & 33 & 36 & 1 & 38 & 48 & 2 & 45 & 24 & 1 \\
\hline Death & $\mathrm{N}$ & $\mathrm{N}$ & $\mathrm{N}$ & $\mathrm{N}$ & $\mathrm{Y}$ & $\mathrm{N}$ & $\mathrm{N}$ & $\mathrm{N}$ & $\mathrm{N}$ & $\mathrm{N}$ & $\mathrm{Y}$ \\
\hline Response to LRN * & Fail & Fail & Fail & NED & Fail & NED & NED & Fail & NED & Fail & Fail \\
\hline
\end{tabular}




\subsection{Samples}

The biospecimens for this study were provided by the Biobank of Seoul St. Mary's Hospital, the Catholic University of Korea. The formalin-fixed, paraffin-embedded (FFPE) samples of 22 paired normal and tumor tissues were obtained from ccRCC patients.

\subsection{Customized NGS Gene Panel}

We developed a customized NGS gene panel for ccRCC with 156 genes. This panel included 123 survival-specific genes newly discovered by machine learning, 21 of the mutated genes ranked above 5\% in TCGA-KIRC (Table S3), and 14 solid tumor-associated genes (Table S4).

\subsection{Library Preparation and Sequencing}

Genomic DNA was extracted from FFPE tissues using a GeneRead DNA FFPE Kit (Cat. No. 180134, Qiagen, Hilden, Germany) according to the manufacturer's instructions. Targeted DNA sequencing libraries were constructed using a SOLIDaccutest-renal cancer panel (Version 1.1, NGeneBio, Seoul, Korea) with $400 \mathrm{ng}$ of genomic DNA. Briefly, genomic DNA was fragmented and tagged to generate adapter-tagged libraries. Each library was indexed by sample and pooled for target enrichment. Target enrichment was performed by hybridization capture using a custom-designed gene panel that targeted the entire coding sequence (CDS, $804 \mathrm{~Kb}$ ) of 156 genes specific to ccRCC. The quality of the libraries was measured with TapeStation 4200 (Version A.02.02., Agilent Technologies, Santa Clara, CA, USA) and quantified using Qubit 4 (Thermo Fisher Scientific, Waltham, MA, USA). Sequencing was performed on an Illumina MiSeq and Nextseq platform (Version 3.1 and Version 4.0, Illumina, San Diego, CA, USA).

\subsection{NGS Data Analysis}

DNA sequencing data were analyzed using a SOLIDaccutest-renal cancer panel customized NGS analysis pipeline (NGeneBio, Seoul, Korea). Briefly, FASTQ files that store the sequence fragments of each sample were aligned to the hig reference genome using an alignment algorithm for aligning sequence reads or assembly contigs against a large reference genome such as the human genome. After read mapping, various post-processes such as duplicate removal, base quality recalibration, and read sorting were performed following the best practices recommended by Genome Analysis Tool Kit (GATK4 (Version 4.0.12.0), Broad Institute, Cambridge, MA, USA) [14]. Single-nucleotide polymorphisms (SNPs) and indel variants were detected using GATK4 Mutect2 (Version 4.0.12.0, Broad Institute, Cambridge, MA, USA) [15], and the variants at Variant allele frequency $>2 \%$, alternate allele count $>5$, and depth $>100$ were included in this study. Variant annotation was performed by a combination of SnpEff and snpSift (Version 4.0, Pablo Cingolani, http: / / pcingola.github.io/SnpEff/, accessed on 15 November 2021) [16]. Nonpathogenic (population frequency $>1 \%$ ) polymorphisms were filtered out using large-scale genome databases such as gnomAD [17] and 1000 Genome [18]. Pathogenicity was determined by the in-house database constructed using databases provide genomic interpretations such as COSMIC [19], ClinVar [20], and various cancer-related literature. Genes that were interpreted as false positives due to poor mapping quality were excluded from the analysis.

\subsection{Dataset}

We gathered data from the Cancer Genome Atlas (TCGA) and Renal Cell CancerEU/FR (RECA-EU) projects for closer scrutiny. They encompass clinical features from each patient including demographics, tumor stage, vital status since the first surgical procedure, and corresponding sequencing reads from their cancer genome, regardless of the class of variant. The TCGA-KIRC dataset can be found at https://www.cbioportal.org/study / summary?id=kirc_tcga (accessed on 7 April 2021), and the renal cell cancer (EU/FR) data from the RECA-EU are available for download at https:/ / dcc.icgc.org/projects/RECA-EU (accessed on 27 May 2021). Both samples originated from renal cell carcinoma, and 451 of 
538 donors from TCGA-KIRC and 422 of 475 donors from RECA-EU were selected from donors with simple somatic mutation to optimize the quality of data analysis. The TCGAKIRC dataset covers clear cell carcinoma only, whereas the RECA-EU data focus on but are not limited to the clear cell subtype. They both use hg19 (GrCh37) annotation. To know the mutation frequency in the normal control population in South Korea, we also had access to data from the Korea Biobank Array Project (referred to as KoreanChip), approved by the National Biobank of Korea, the Centers for Disease Control and Prevention, Republic of Korea (KBN-2019-019, approval date: 21 March 2019). The Korea Biobank Array Project was initiated in 2014 by the Korea National Institute of Health and included 210,000 participants aged 40-69 years via the Korean Genome and Epidemiology Study [21] to implement a customized Korean genome structure-based array with high genomic coverage and abundant functional variants of low to rare frequency [22]. The KoreanChip comprised $>833,000$ markers including $>247,000$ rare-frequency or functional variants estimated from $>2500$ sequencing data in Koreans. Of the 833,000 markers, 208,000 functional markers were genotyped. Particularly, >89,000 markers were present in East Asians.

\subsection{Data Preparation}

For further clarification of our findings, we divided the donors obtained from TCGAKIRC and RECA-EU datasets into two independent groups to exam the propensity for OS and DFS: the group with mutations in the selected genes most likely to contribute to renal cancer and the control group. To investigate the relationships between clinical characteristics and the mutated genes, we extracted data on six distinctive clinical variables: age, sex, tumor stage, metastasis, survival time, and recurrence. The categorical variables from clinical data were one-hot encoded; indicated by binary values, the rest were used as ordinal, and the unit of survival time was months. The raw data from the RECA-EU containing Ensembl IDs were converted to hg19 RefSeq annotation before mapping them into the gene list from the TCGA-KIRC. The raw format of KoreanChip data was processed using PLINK (Version 1.9, Shaun Purcell and Christopher Chang, www.cog-genomics.org/plink/1.9/, accessed on 15 November 2021). Genomic variants were genotyped from SNPs using Ensembl Variant Effect Predictor (VEP) (Version 96, Sarah E Hun (2019), http: / / apr2019.archive.ensembl.org/index.html, accessed on 15 November 2021), and additional identifiers were retrieved using the biomaRt package from R (Version 3.5, Durinck S, https: / / bioconductor.org/packages/release/bioc/html/biomaRt.html, accessed on 15 November 2021).

\subsection{Statistical Analysis}

To find the genes most strongly associated with renal cancer, a Pearson correlation analysis was performed to validate the associations between the clinical variables and the genes with clinicopathological importance. This method was also applied to evaluate the strength of association with the survival-related factors and to plot the correlation matrix table. The correlation coefficients, denoted by Pearson's $R$, were measured by the function cor in the Stats R-Package (RStudio Team (2016), Boston, MA, USA, http: / / www.rstudio.com, accessed on 15 November 2021), and any coefficient greater than 0.3 was re-evaluated using Spearman's test. When performing survival analyses using datasets from TCGA-KIRC and RECA-EU, the Kaplan-Meier method and the multivariate Cox proportional hazard model were used. The survival curves and hazard ratios over the given survival periods were estimated by utilizing a Python library called lifelines (0.26.4 version, Cameron Davidson-Pilon, https://lifelines.readthedocs.io/en/latest/). All pairs of survival curves were compared using the log-rank test module from lifeline statistics to confirm the discrepancy between groups. During the analysis, statistical significance was determined with $p<0.05$ as a cutoff value and with a $95 \%$ confidence level. 


\section{Results}

We developed a customized NGS gene panel of 156 genes related to ccRCC, including 123 survival-specific genes, and performed NGS analysis on samples from 22 patients. Of the 156 genes, 37 were mutated with a total of 100 mutations (data not shown). Of the 37 genes, 16 were in the top $5 \%$ or more, and 21 survival-specific genes were identified. The steps we performed, from gene discovery to NGS data analysis, are illustrated in Figure 1.

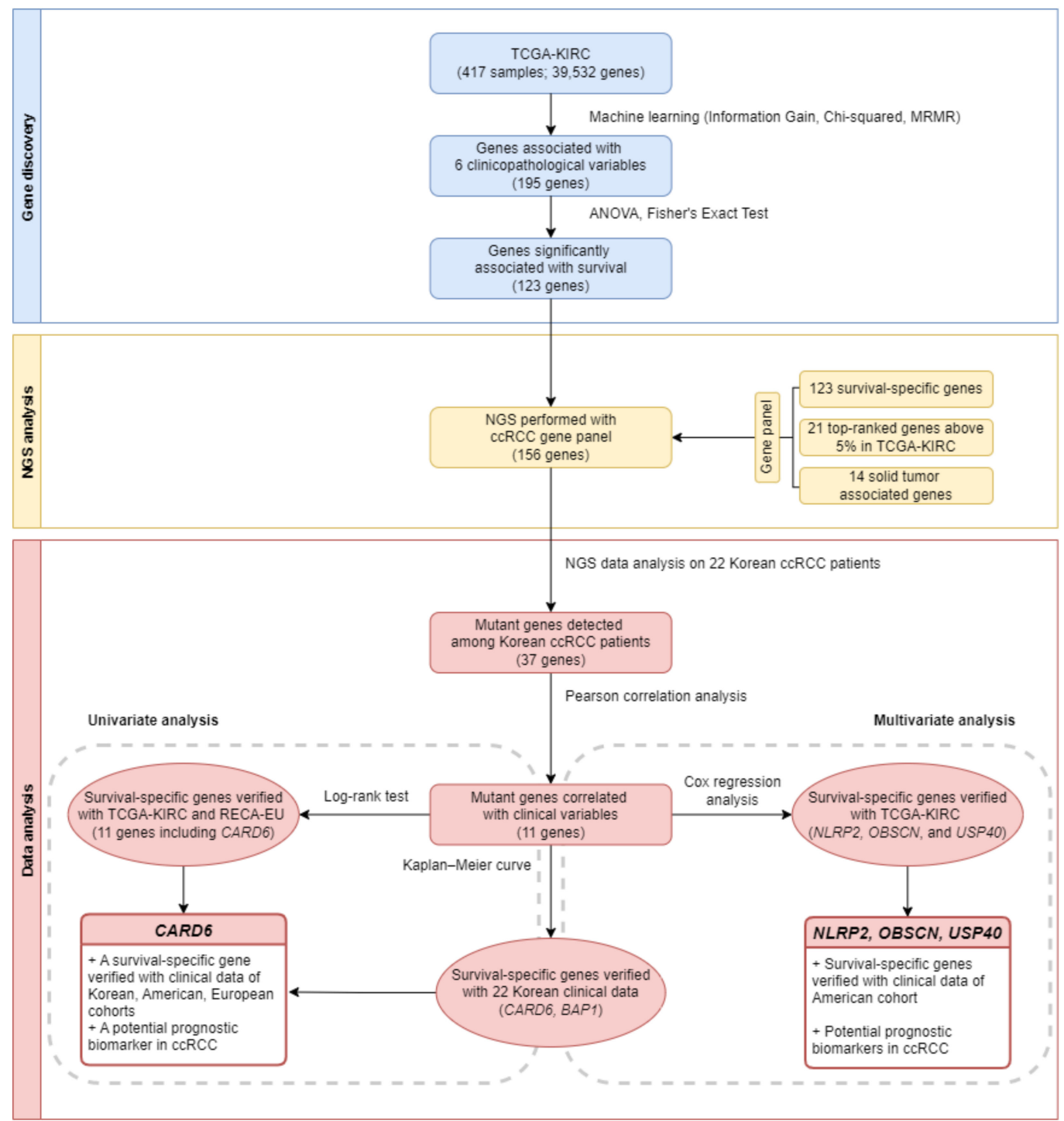

Figure 1. Workflow of the study. NGS, Next Generation Sequencing; TCGA-KIRC, The Cancer Genome Atlas Kidney Renal Clear Cell Carcinoma; MRMR, Minimum Redundancy and Maximum Relevance; ANOVA, Analysis of variance; ccRCC, clear cell renal cell carcinoma; RECA-EU, Renal Cell Cancer-European Union.

The clinicopathological findings of 22 ccRCC patients are summarized in Table 1 . The patients were 15 men and seven women with a mean age of 59.5 years (33-82 years). Their mean tumor size was $6.6 \mathrm{~cm}(2.5-14.5 \mathrm{~cm})$. The prevalence of nuclear grades I, II, III, and IV were $0(0 \%), 6(27.3 \%), 11(50 \%)$, and $5(22.7 \%)$, respectively. The prevalence of TNM stages was as follows. Tumor stage: seven patients $(31.8 \%)$ with T1, five $(22.7 \%)$ with T2, and $10(45.5 \%)$ with T3. Lymph node staging showed 21 patients (95.5\%) with N0 and one (4.5\%) with N1. Distant metastasis staging indicated 12 patients (54.5\%) with M0 and 10 (45.5\%) with M1. Metastases were found in 10 of $22(45.5 \%)$ ccRCC patients, all of which 
occurred in the lung and also in other sites such as the bones $(3 / 22,13.6 \%)$, brain $(2 / 22$, $9.1 \%)$, abdomen $(1 / 22,4.5 \%)$, liver $(2 / 22,9.1 \%)$, retroperitoneum $(1 / 22,4.5 \%)$, jejunum $(1 / 22,4.5 \%)$, and peritoneum $(1 / 22,4.5 \%)$. The mean OS of the patients was 34.6 months (12-50 months), and the mean DFS was 22.9 months (0-48 months). Of the 22 patients who underwent LRN, 12 (54.5\%) showed no evidence of disease, and the remaining $10(45.5 \%)$ had recurrence or metastasis due to treatment failure. Four of 22 patients $(18.2 \%)$ died of ccRCC, and recurrence and metastasis were observed in all of them. The frequencies of the top 16 mutant genes found in this study are shown in Table 2. The mutation frequency of each gene in our study was compared with data from other studies, and the VHL gene was found to have the highest frequency. The frequencies of four genes (VHL, SETD2, TSHZ3, and SPEN) were particularly high $(91 \%, 50 \%, 14 \%$, and $18 \%$, respectively) compared to other studies such as TCGA $(54 \%, 21 \%, 6 \%$, and 5\%), RECA-EU $(61 \%, 22 \%, 8 \%$, and 5\%), Tokyo (41\%, 11\%, 2.8\%, and 0), and Taiwan (50\%, 22\%, unknown, and unknown) [10]. Our results also differed from other studies in terms of mutation frequency. In particular, mutations in VHL were found in 20 (91\%) patients, which was noticeably high when compared to other results. All VHL gene mutations in 20 ccRCC patients were different, indicating that each tumor was highly heterogeneous genetically. Of the VHL mutations identified, 16 variants were reported to COSMIC, and the remaining four variants were not reported. In ClinVar, 10 variants were reported as pathogenic, two as likely pathogenic, one as of uncertain significance, and seven as unknown (Table 3).

Table 2. Comparison of top-ranked mutated genes in clear cell renal cell carcinoma in 22 Korean patients and patients from other regions.

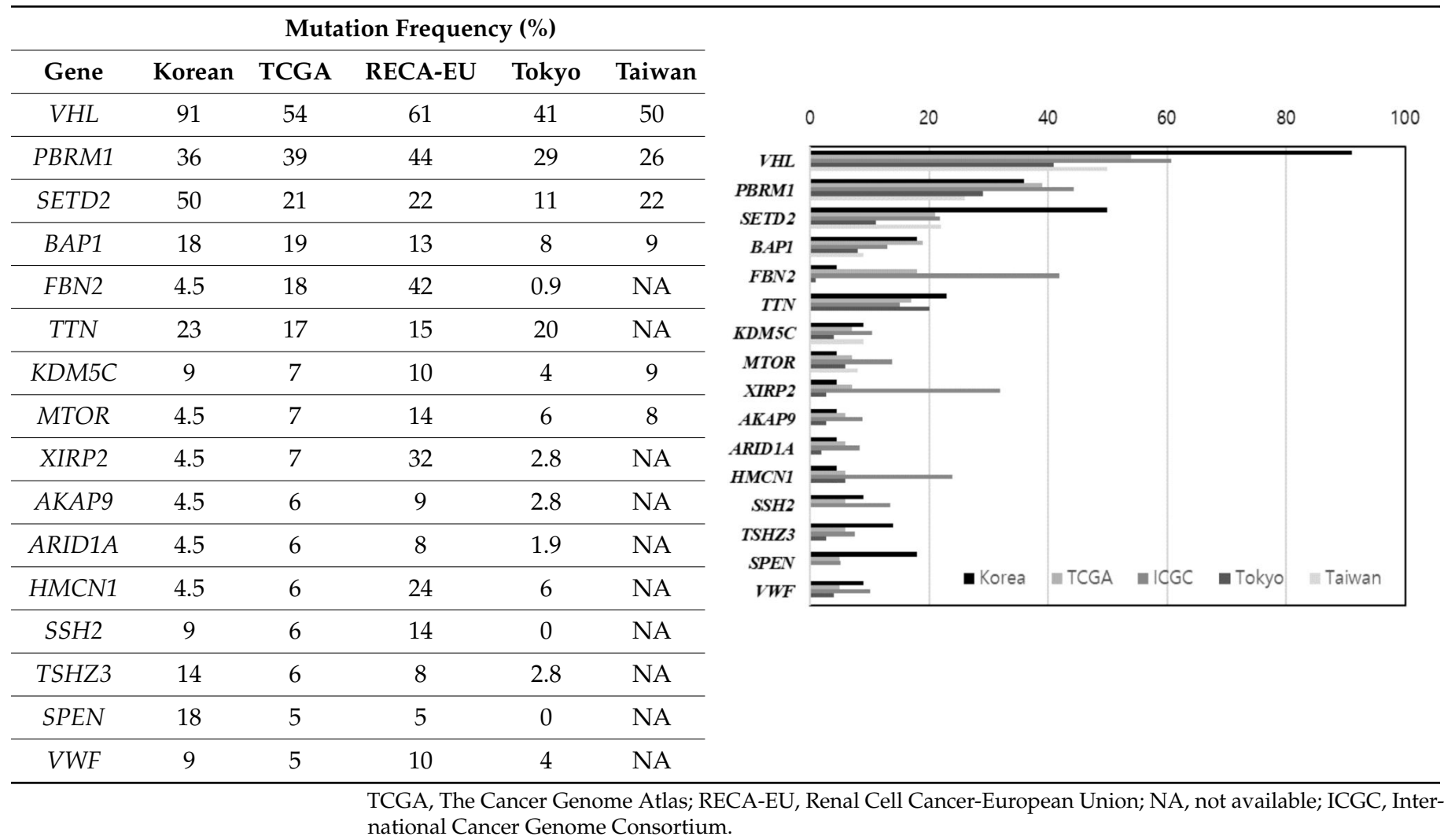


Table 3. Types of mutations in VHL genes found in 22 Korean patients with clear cell renal cell carcinomas.

\begin{tabular}{|c|c|c|c|c|c|}
\hline HGVS.c (cDNA) & $\begin{array}{l}\text { HGVS.p } \\
\text { (Protein) }\end{array}$ & $\begin{array}{l}\text { HGVS.p } \\
\text { (Single) }\end{array}$ & Variant Type & ClinVar & COSMIC ID \\
\hline c. $337 \mathrm{C}>\mathrm{T}$ & p.Arg113 & R113 & Stop gained & Pathogenic & COSM30228 \\
\hline c.353T $>C$ & p.Leu118Pro & L118P & Missense variant Intron variant & Pathogenic & COSM14312 \\
\hline c.174_208delGCCGC & & & & & \\
\hline GGCCCGTG CTGCG & p.Pro59fs & P59fs & Frameshift variant & Unknown & - \\
\hline \multicolumn{6}{|l|}{ CTCGGTGAACTCG CGCG } \\
\hline c. $463+1 G>A$ & - & - & Intron variant & Pathogenic & COSM51391 \\
\hline c. $263 \mathrm{G}>\mathrm{A}$ & p.Trp88 & W88 & Stop gained & Pathogenic & COSM18070 \\
\hline $\begin{array}{c}\text { c.220_231dupGTCAT } \\
\text { CTTCTGC }\end{array}$ & p.Val74_Cys77dup & V74_C77dup & Conservative inframe insertion & Unknown & - \\
\hline c. $257 \mathrm{C}>\mathrm{T}$ & p.Pro86Leu & P86L & Missense variant & Pathogenic & COSM18028 \\
\hline c. $473 \mathrm{~T}>\mathrm{A}$ & p.Leu158Gln & L158Q & Missense variant & Likely pathogenic & COSM14368 \\
\hline c.227_229delTCT & p.Phe76del & F76del & Disruptive inframe deletion & Unknown & COSM53186 \\
\hline c. $430 \mathrm{G}>\mathrm{T}$ & p.Gly144 & G144 & Stop gained Intron variant & Pathogenic & COSM25682 \\
\hline c.449delA & p.Asn $150 \mathrm{fs}$ & N150fs & Frameshift variant Intron variant & Unknown & COSM17843 \\
\hline c. $332 \mathrm{G}>\mathrm{T}$ & p.Ser111Gly & S1111 & Missense variant & $\begin{array}{l}\text { Uncertain } \\
\text { significance }\end{array}$ & COSM36341 \\
\hline c.280delG & p.Glu94fs & E94fs & Frameshift variant & Unknown & - \\
\hline c. $281 \mathrm{~A}>\mathrm{T}$ & p.Glu94Val & E94V & Missense variant & Unknown & - \\
\hline c. $331 \mathrm{~A}>\mathrm{G}$ & p.Ser111Gly & S111G & Missense variant & Pathogenic & COSM18353 \\
\hline c. $337 \mathrm{C}>\mathrm{T}$ & p.Arg113 & $\mathrm{R} 113$ & Stop gained & Pathogenic & COSM30228 \\
\hline c. $266 \mathrm{~T}>\mathrm{A}$ & p.Leu89His & $\mathrm{L} 89 \mathrm{H}$ & Missense variant & Likely pathogenic & COSM14305 \\
\hline c.523dupT & p.Tyr175fs & Y175fs & Frameshift variant & Unknown & COSM253386 \\
\hline c. $203 \mathrm{C}>\mathrm{A}$ & p.Ser68 & S68 & Stop gained & Pathogenic & COSM14372 \\
\hline c. $362 A>G$ & p.Asp121Gly & D121G & Missense variant intron variant & Pathogenic & COSM18009 \\
\hline
\end{tabular}

The associations between the mutated genes in 22 Korean patients with ccRCC and their corresponding clinicopathological factors are shown in Table 4 . A total of 37 mutated genes was found, among which only 11 (ADAMTS10, CARD6, NLRP2, OBSCN, SECISBP2L, USP40, AKAP9, ARID1A, BAP1, KDM5C, and SETD2) showed correlations with various clinicopathological findings. The 11 genes included six of the 123 survival-specific genes and five of the top 21 genes in TCGA-KIRC. Furthermore, we examined the mutation frequencies of the 11 genes listed in Table 4 in the normal population using Koreanchip, which gives the genomic sequencing reads of 210,000 normal Koreans [21,22]. As a result of our population-based analysis, mutations in 11 genes found in ccRCC patients were not found in Koreans without ccRCC.

Table 4. Eleven clinicopathologically significant mutated genes found in 22 Korean patients with clear cell renal cell carcinoma.

\begin{tabular}{|c|c|c|c|c|c|c|c|c|c|c|c|c|}
\hline \multirow{2}{*}{$\begin{array}{l}\text { Clinical } \\
\text { Variable }\end{array}$} & \multirow{2}{*}{ Result } & \multicolumn{6}{|c|}{ Survival-Specific Mutated Genes of ccRCC } & \multicolumn{5}{|c|}{ Top Ranked Mutated Genes of TCGA_KIRC } \\
\hline & & ADAMTS10 & CARD6 & NLRP2 & OBSCN & SECISBP2L & USP40 & AKAP9 & ARID1A & BAP1 & KDM5C & SETD2 \\
\hline Overall & $r$ & 0.287 & $-0.441 *$ & -0.055 & 0.119 & 0.159 & -0.055 & -0.055 & 0.287 & -0.235 & -0.204 & 0.377 \\
\hline Survival & $p$-value & 0.195 & 0.04 & 0.806 & 0.597 & 0.481 & 0.806 & 0.806 & 0.195 & 0.291 & 0.361 & 0.084 \\
\hline Disease Free & $r$ & 0.350 & -0.124 & -0.180 & -0.234 & -0.180 & -0.180 & -0.180 & -0.350 & $-0.465 *$ & -0.221 & -0.115 \\
\hline Survival & $p$-value & 0.110 & 0.581 & 0.422 & 0.295 & 0.422 & 0.422 & 0.422 & 0.110 & 0.029 & 0.323 & 0.610 \\
\hline \multirow{2}{*}{ Death } & $r$ & -0.087 & -0.087 & -0.087 & -0.126 & 0.463 * & -0.087 & -0.087 & -0.087 & 0.389 & -0.126 & 0.024 \\
\hline & $p$-value & 0.701 & 0.701 & 0.701 & 0.578 & 0.03 & 0.701 & 0.701 & 0.701 & 0.073 & 0.577 & 0.916 \\
\hline Nuclear & $r$ & -0.299 & 0.019 & 0.019 & 0.034 & 0.318 & 0.019 & 0.019 & -0.299 & 0.192 & 0.244 & 0.451 * \\
\hline Grade & $p$-value & 0.176 & 0.934 & 0.934 & 0.881 & 0.149 & 0.934 & 0.934 & 0.176 & 0.392 & 0.274 & 0.035 \\
\hline \multirow{2}{*}{$\begin{array}{l}\text { Sarcomatoid } \\
\text { component }\end{array}$} & $r$ & -0.069 & -0.069 & $0.690^{* *}$ & $0.474 *$ & -0.069 & $0.690 * *$ & $0.690 * *$ & -0.069 & 0.261 & -0.1 & 0.314 \\
\hline & $p$-value & 0.76 & 0.76 & 0.00038 & 0.026 & 0.76 & 0.00038 & 0.00038 & 0.76 & 0.241 & 0.658 & 0.155 \\
\hline \multirow{2}{*}{ N-stage } & $r$ & -0.048 & -0.048 & -0.048 & -0.069 & $1.000 * *$ & -0.048 & -0.048 & -0.048 & -0.103 & $0.690^{* *}$ & 0.217 \\
\hline & $p$-value & 0.833 & 0.833 & 0.833 & 0.761 & $2.20 \mathrm{e}-16$ & 0.833 & 0.833 & 0.833 & 0.649 & 0.00038 & 0.333 \\
\hline \multirow{2}{*}{ Sex } & $r$ & -0.149 & -0.149 & -0.149 & $0.462 *$ & -0.149 & -0.149 & -0.149 & -0.149 & 0.184 & -0.216 & -0.379 \\
\hline & $p$-value & 0.508 & 0.508 & 0.508 & 0.03 & 0.508 & 0.508 & 0.508 & 0.508 & 0.412 & 0.334 & 0.082 \\
\hline \multirow{2}{*}{ Tumor size } & $r$ & $0.585^{* *}$ & -0.104 & -0.082 & -0.119 & 0.252 & -0.082 & -0.082 & $0.585^{* *}$ & -0.169 & 0.107 & 0.123 \\
\hline & $p$-value & 0.004 & 0.645 & 0.717 & 0.599 & 0.259 & 0.717 & 0.717 & 0.004 & 0.453 & 0.636 & 0.585 \\
\hline
\end{tabular}

* is used for $p$-values $<0.05 . * *$ are used for $p$-values $<0.001$. Statistical significant values are bolded. The $p$-values are from Pearson's correlation analysis.

A gene associated with OS, CARD6 ( $p=0.04, r=-0.441)$, was the only one identified among 123 survival-specific mutant genes. The Pearson's correlation test revealed that 
the presence of mutations in CARD6 was inversely related to the period of OS (Table S5). Patient P10, with CARD6 mutations, had an OS of 14 months, which was the shortest of the 22 patients. The gene found to be associated with DFS ( $p=0.029, r=-0.465)$ was $B A P 1$. The Pearson's correlation test showed that the presence of the BAP1 mutation resulted in shorter DFS (Table S6). The presence of mutation in SECISBP2L showed an association with mortality $(p=0.03, r=0.463)$ (Table S7). The gene SETD2 showed an association with nuclear grade $(p=0.035, r=0.451)$; the higher the grade, the more frequent the mutation (Table S8). Genes associated with the sarcomatoid component were NLRP2 $(p=0.00038, r=0.690)$, OBSCN $(p=0.026, r=0.474)$, USP40 $(p=0.00038, r=0.690)$, and AKAP9 ( $p=0.00038, r=0.690)$ (Tables S9-12). In the presence of a sarcomatoid component in histological findings, mutations in one or more of these four genes increased. Patients with mutations in NLRP2, USP40, or AKAP9 had a strong association with sarcomatoid components, and patients with mutations in OBSCN showed a moderate correlation with sarcomatoid components. The mutated genes associated with lymph node metastasis were SECISBP2L $(p=2.20 \mathrm{e}-16, r=1.000)$ and KDM5C $(p=0.00038, r=0.690)$; both of these showed a very strong association (Tables S7 and S13). The Pearson's correlation test revealed that the presence of mutations in SECISBP2L was closely related to the spread of cancer cells to the lymph nodes and also showed a significant association with mortality $(p=0.03, r=0.463)$. Mutations in OBSCN were moderately associated with sex $(p=0.03$, $r=0.462)$ as both patients with OBSCN mutations were female (P6 and P12) (Table S10). The genes significantly associated with tumor size were ADAMTS10 $(p=0.004, r=0.585)$ and ARID1A ( $p=0.004, r=0.585)$ (Tables S14 and S15). ADAMTS10 and ARID1A mutations were observed simultaneously in the patient (P18) who had the largest tumor size $(14.5 \mathrm{~cm})$ among the 22 patients (size of tumors without mutation, mean: $6.2 \mathrm{~cm}$, median: $5.5 \mathrm{~cm}$ ).

We identified the types and frequencies of mutations in 11 clinicopathologically significant genes in 22 Korean ccRCC patients and compared them with those reported by TCGA-KIRC and RECA-EU (Table 5). In this study, a mutation in CARD6 (missense_variant; c. $2674 \mathrm{G}>\mathrm{A}$, p.Gly892Arg) was found in 1 of 22 patients $(4.5 \%, \mathrm{P} 10)$. This was identified as the only survival-specific gene. OBSCN mutations were observed in 2 of 22 patients (9.1\%), both of whom were female. Mutations in both ADAMTS10 and ARID1A genes were found in patient P18. The tumor in this patient was $14.5 \mathrm{~cm}$, the largest among the 22 patients. Therefore, if there is a mutation in these two genes, the size of the tumor is expected to be large. In addition, various types of SETD2 mutations (missense, frameshift, stop gained, splice variant, intron variant, and in-frame deletion) were observed, and the mutation frequency was 50\%, more than double that reported in TCGA (21\%) or RECA-EU $(20 \%)$.

Table 6 compares the associations of survival rates obtained from TCGA-KIRC (Firehose Legacy and PanCancer Atlas) and RECA-EU with the 11 clinicopathologically significant genes. Analysis of the TCGA-KIRC cohort confirmed that all six survival-specific genes were significantly associated with survival rate, OS, and/or DFS in ccRCC patients. However, in the RECA-EU data, only four genes (ADAMTS10, CARD6, OBSCN, and USP40) showed a significant association with survival rate. In addition, the top-ranked mutated genes showed little correlation with survival rate in the TCGA data, and only SETD2 showed a significant association with DFS. However, in the RECA-EU data, the top-ranked mutant genes showed a correlation with survival rate, OS, and/or DFS in all five genes. 
Table 5. Types and frequencies of 11 clinically significant mutated genes found in 22 Korean patients with clear cell renal cell carcinoma.

\begin{tabular}{|c|c|c|c|c|c|c|c|}
\hline Genes & Mutant Type & $\begin{array}{l}\text { Mutation } \\
\text { HGVS.c }\end{array}$ & HGVS.p & Mutation Frequency \% & Patient ID & $\begin{array}{l}\text { TCGA-KIRC (Firehose) } \\
\text { Mutation Frequency } \%\end{array}$ & $\begin{array}{l}\text { RECA-EU Mutation } \\
\text { Frequency } \%\end{array}$ \\
\hline ADAMTS10 & missense_variant & c. $2303 \mathrm{G}>\mathrm{A}$ & p.Arg768His & 4.5 & P18 & 0.9 & 2.1 \\
\hline CARD6 & missense_variant & c. $2674 \mathrm{G}>\mathrm{A}$ & p.Gly892Arg & 4.5 & P10 & 1.1 & 0.7 \\
\hline NLRP2 & missense_variant & c. $2233 \mathrm{C}>\mathrm{T}$ & p.Arg745Trp & 4.5 & P05 & 1.1 & 4.7 \\
\hline \multirow[t]{2}{*}{ OBSCN } & missense_variant & c. $3529 \mathrm{C}>\mathrm{G}$ & p.Gln1177Glu & 9.1 & P12 & 1.1 & 6 \\
\hline & & c.12072_12073delTCinsCT & p.Arg4025Cys & & P12 & & \\
\hline SECISBP2L & missense_variant & c. $1930 A>G$ & p.Met644Val & 4.5 & P11 & 1.8 & 3.5 \\
\hline USP40 & frameshift_variant & c.3477dupA & p.Gln1160fs & 4.5 & P05 & 2.2 & 4.5 \\
\hline AKAP9 & missense_variant & c. $89 \mathrm{~A}>\mathrm{T}$ & p.Gln30Leu & 4.5 & P05 & 6 & 7 \\
\hline ARID1A & $\begin{array}{l}\text { missense_variant and } \\
\text { splice_region_variant }\end{array}$ & c. $437 \mathrm{G}>\mathrm{C}$ & p.Arg146Thr & & P08 & & \\
\hline \multirow[t]{2}{*}{$B A P 1$} & frameshift_variant & $\begin{array}{c}\text { c.878dupC } \\
\text { c.1636_1642delTACAACC }\end{array}$ & $\begin{array}{l}\text { p.Leu294fs } \\
\text { p.Tyr546fs }\end{array}$ & 18.1 & $\begin{array}{l}\text { P12 } \\
\text { P16 }\end{array}$ & 19 & 13 \\
\hline & $\begin{array}{l}\text { splice_acceptor_variant and } \\
\text { splice_region_variant }\end{array}$ & c. $581-2 \mathrm{~A}>\mathrm{G}$ & Unknown & & $\mathrm{P} 22$ & & \\
\hline \multirow{5}{*}{ KDM5C } & frameshift_variant & c.3460delG & p.Glu1154fs & 91 & P10 & 7 & 10 \\
\hline & $\begin{array}{l}\text { splice_donor_variant } \\
\text { \&intron variant }\end{array}$ & c. $531+2 \mathrm{~T}>\mathrm{A}$ & Unknown & & P11 & & \\
\hline & & c.2357_2358delGCinsTT & p.Cys786Phe & & P08 & & \\
\hline & & c. $577 \mathrm{C}>\mathrm{T}$ & p.Pro193Ser & & P22 & & \\
\hline & frameshift_variant & c.7537_7546dupACTCACGGTG & p.Val2516fs & & P11 & & \\
\hline \multirow[t]{6}{*}{ SETD2 } & & c. $572 \mathrm{delC}$ & p.Pro191fs & 50 & P22 & 21 & 20 \\
\hline & stop_gained & c. $6520 \mathrm{C}>\mathrm{T}$ & p.Gln2174 & & P05 & & \\
\hline & & c. $4486 \mathrm{C}>\mathrm{T}$ & p.Arg1496 & & P21 & & \\
\hline & $\begin{array}{l}\text { stop_gained and } \\
\text { splice_region_variant }\end{array}$ & c.5013T $>\mathrm{G}$ & p.Tyr1671 & & P12 & & \\
\hline & $\begin{array}{l}\text { splice_donor_variant and } \\
\text { intron variant }\end{array}$ & c. $4715+1 G>A$ & Unknown & & P03 & & \\
\hline & conservative_inframe_deletion & $\begin{array}{l}\text { C.625_681delACAGA- } \\
\text { GCCAGTGGCCTTG- } \\
\text { CCACATACACCAAT- } \\
\text { AACAGTTCTAATG- } \\
\text { GCAGCACCAGTA }\end{array}$ & p.Thr209_Val227del & & P20 & & \\
\hline
\end{tabular}

TCGA-KIRC, The Cancer Genome Atlas Kidney Renal Clear Cell Carcinoma; RECA-EU, Renal Cell Cancer-European Union; P, patient. 
Table 6. Comparison of survival analysis of TCGA-KIRC and RECA-EU data for 11 clinicopathologically significant mutated genes found in 22 Korean patients with clear cell renal cell carcinoma.

\begin{tabular}{|c|c|c|c|c|c|c|c|c|c|c|c|c|}
\hline \multirow{2}{*}{\multicolumn{2}{|c|}{ Survival Analysis }} & \multicolumn{6}{|c|}{ Survival-Specific Mutated Genes of ccRCC } & \multicolumn{5}{|c|}{ Top Ranked Mutated Genes of TCGA_KIRC } \\
\hline & & ADAMTS10 & CARD 6 & NLRP2 & OBSCN & SECISBP2L & USP40 & AKAP9 & ARID1A & BAP1 & KDM5C & SETD2 \\
\hline \multirow{5}{*}{ TCGA_KIRC } & Overall Survival & & & & & & & & & & & \\
\hline & Firehose Legacy & 0.209 & 0.15 & $0.0029 *$ & $0.0096^{*}$ & 0.112 & 0.378 & 0.284 & 0.906 & 0.577 & 0.102 & 0.821 \\
\hline & PanCancer Atlas & $0.0186^{*}$ & $0.0003^{* *}$ & $0.0142 *$ & 0.289 & 0.0005 ** & 0.53 & 0.845 & 0.464 & 0.0754 & 0.0664 & 0.273 \\
\hline & $\begin{array}{l}\text { Disease free Survival } \\
\text { Firehose Legacy }\end{array}$ & 0.0004 * & 0.074 & $3.09 \mathrm{e}-12 * *$ & $0.0048 *$ & $0.0489 *$ & $0.0003 * *$ & 0.8 & 0.384 & 0.285 & 0.0911 & 0.238 \\
\hline & PanCancer Atlas & NA & NA & $<10^{-10} * *$ & 0.695 & NA & 0.0269 * & 0.177 & 0.228 & 0.401 & 0.488 & 0.0201 * \\
\hline \multirow[b]{2}{*}{ RECA-EU } & Overall Survival & 0.326 & $0.0005 * *$ & 0.061 & 0.423 & 0.564 & $0.042 *$ & 0.047 * & $0.0204 *$ & 0.0024 * & 0.022 * & 0.056 \\
\hline & Disease Free Survival & $0.0011 *$ & 1.0 & 0.65 & $3.28 \mathrm{e}-06$ ** & 0.669 & 0.669 & 0.263 & $1.24 \mathrm{e}-05$ ** & 0.421 & 0.491 & $0.0002 * *$ \\
\hline
\end{tabular}

${ }^{*}$ is used for $p$-values $<0.05 .{ }^{* *}$ are used for $p$-values $<0.001$. Statistical significant values are bolded. The $p$-values are from the Log rank test. ccRCC, clear cell renal cell carcinoma; TCGA_KIRC, The Cancer Genome Atlas Kidney Renal Clear Cell Carcinoma; RECA-EU, Renal Cell Cancer-European Union; NA, Not available.

The six survival-specific mutated genes (ADAMTS10, CARD6, NLRP2, OBSCN, SECISBP2L, and USP40) identified in TCGA-KIRC (Firehose Legacy and PanCancer Atlas) are shown in the survival graphs (Figure 2). Individual survival graphs showed that patients with mutations in each of the six genes had lower survival rates than patients without mutations for OS ( $p=0.0186, p=0.0003, p=0.0029, p=0.0096, p=0.0005$, and $p=0.378)$ and DFS $(p=0.0004, p=0.074, p=3.09 \mathrm{e}-12, p=0.0048, p=0.0489$, and $p=0.0003)$. In addition, Figure 3 shows the survival graphs according to the presence or absence of mutations in all six survival-specific genes in TCGA-KIRC. Patients with mutations in at least one of the six survival-specific genes had lower rates for both OS (Firehose Legacy; $p=0.004$, PanCancer Atlas; $p=3.166 \mathrm{e}-6$ ) and DFS (Firehose Legacy; $p=5.65 \mathrm{e}-9$, PanCancer Atlas; $p=0.0025$ ) than those without mutations.

(a) Overall Survival

ADAMTS10

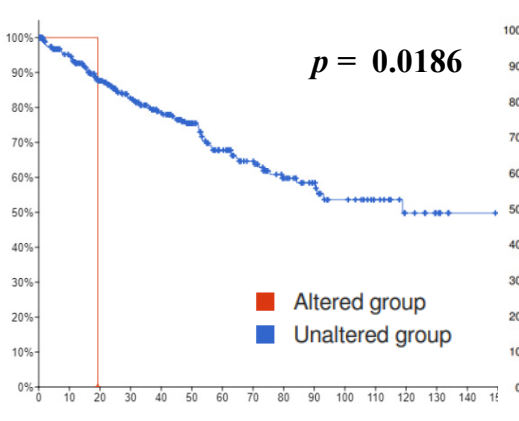

OBSCN

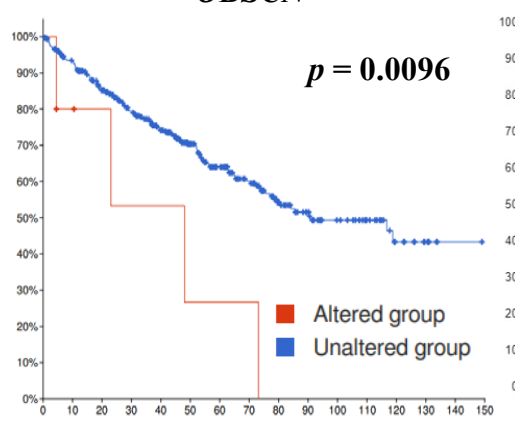

CARD6

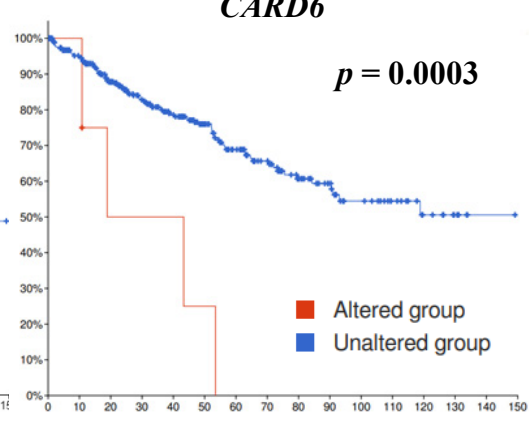

SECISBP2L

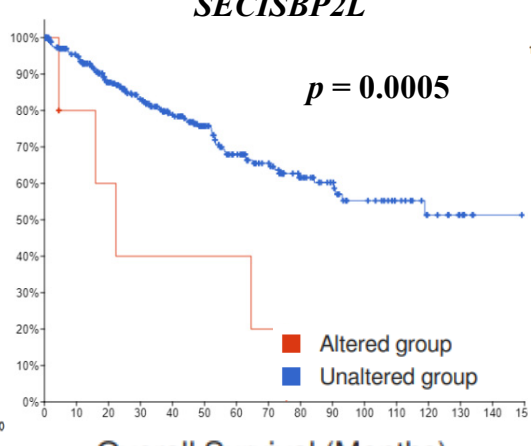

Overall Survival (Months)
NLRP2

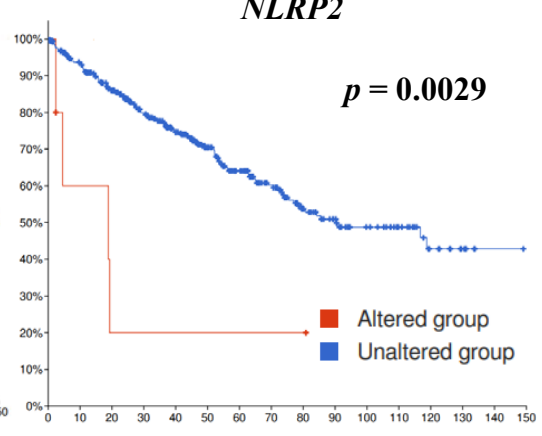

USP40

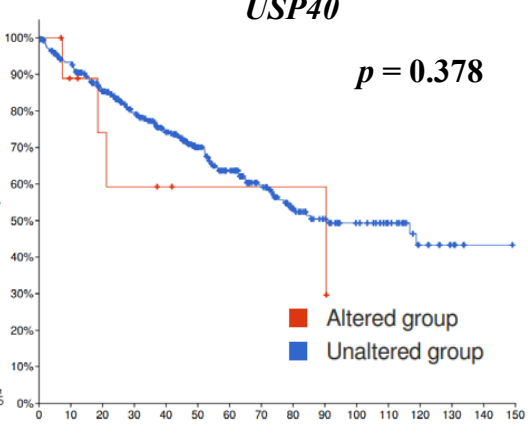

Figure 2. Cont. 


\section{(b) Disease Free Survival}

ADAMTS10

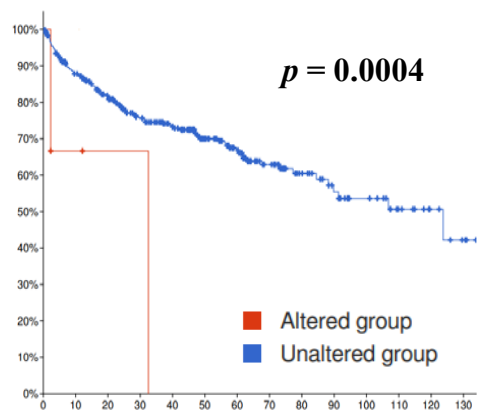

OBSCN

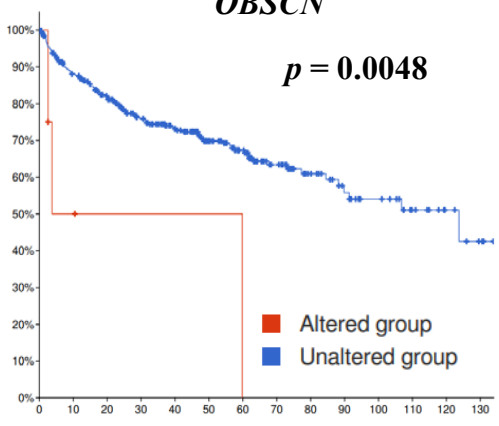

CARD6

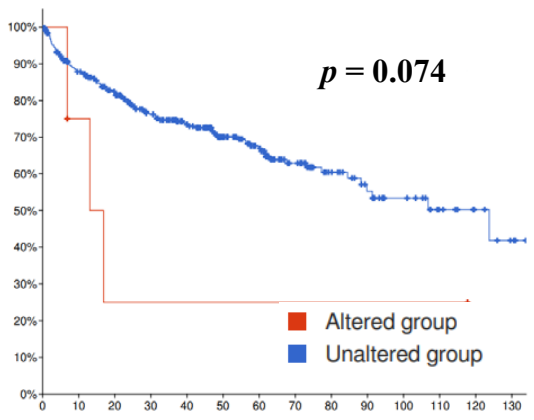

SECISBP2L

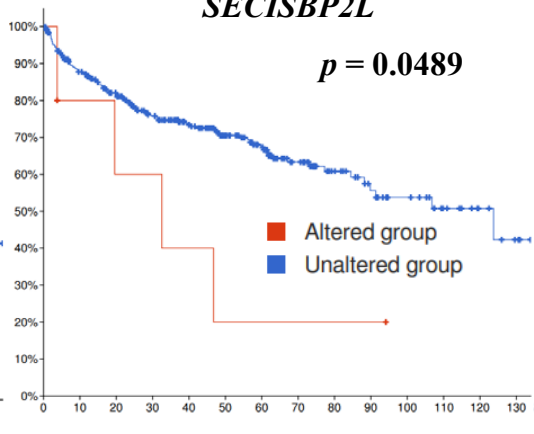

$N L R P 2$

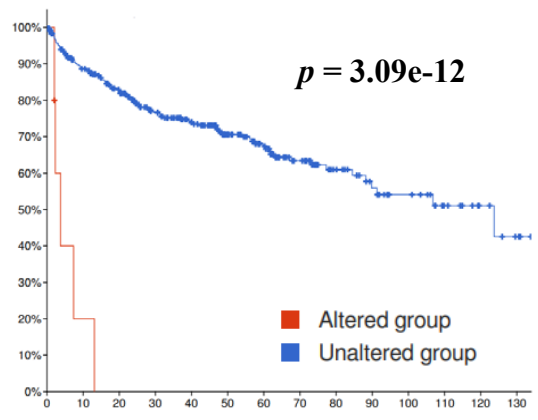

USP40

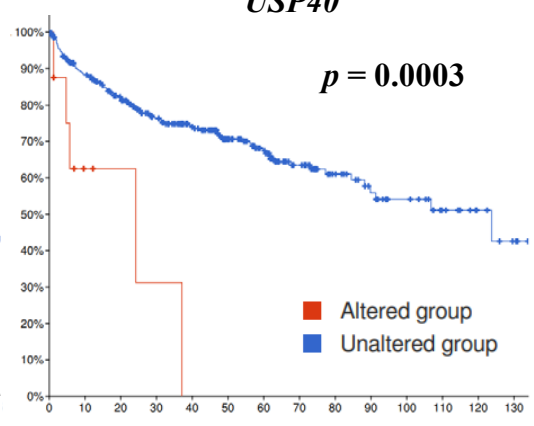

Disease Free (Months)

Figure 2. Survival analysis of TCGA_KIRC data for six survival-specific mutated genes. (a) Overall survival graphs of ADAMTS10, CARD6, and SECISBP2L were obtained from the PanCancer Atlas; overall survival graphs of NLRP2, OBSCN, and USP40 were obtained from TCGA, Firehose Legacy. (b) Disease-free survival graphs of ADAMTS10, CARD6, NLRP2, OBSCN, SECISBP2L, and USP40 were based on data from TCGA, Firehose Legacy.

(a)
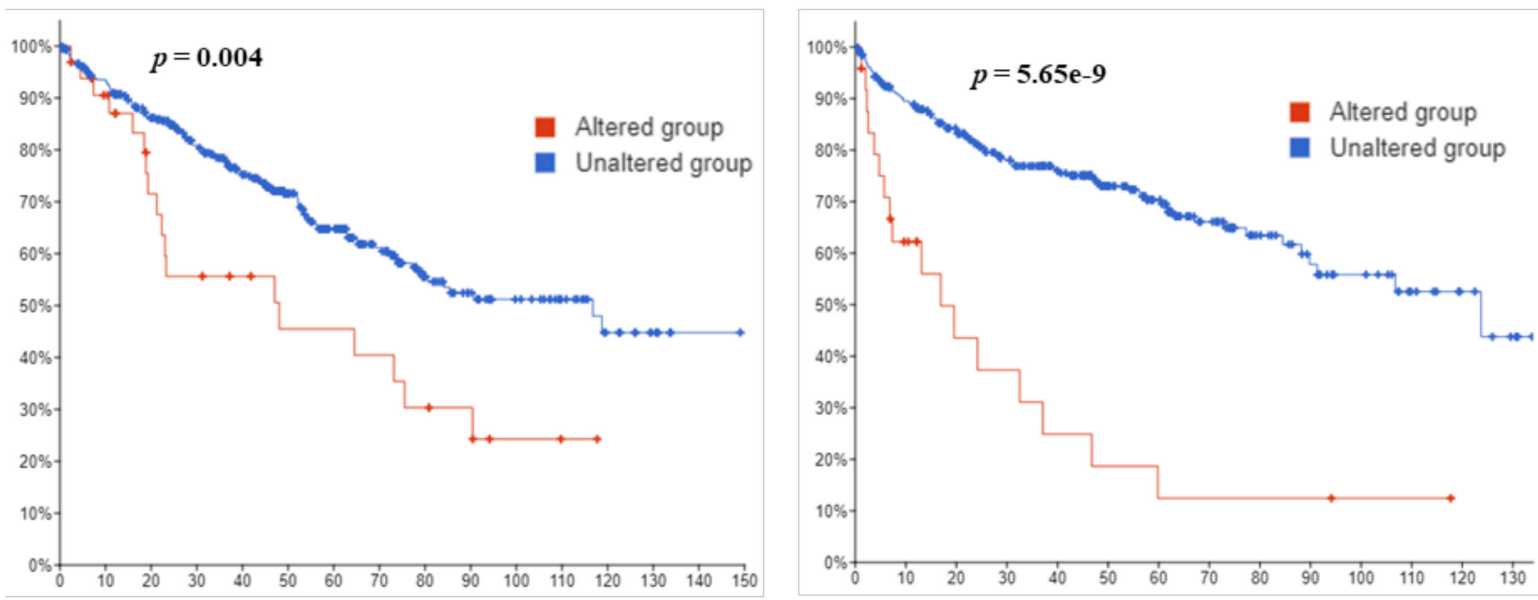

Overall Survival (Months)

\begin{tabular}{c|c|c|}
\hline & $\begin{array}{c}\text { Number of Cases, } \\
\text { Total }\end{array}$ & Number of Events \\
\hline Altered group & 32 & 18 \\
\hline Unaltered group & 416 & 134 \\
\hline
\end{tabular}

Disease Free (Months)

\begin{tabular}{c|c|c}
\hline & $\begin{array}{c}\text { Number of Cases, } \\
\text { Total }\end{array}$ & Number of Events \\
\hline Altered group & 24 & 17 \\
\hline Unaltered group & 340 & 95 \\
\hline
\end{tabular}

Figure 3. Cont. 
(b)
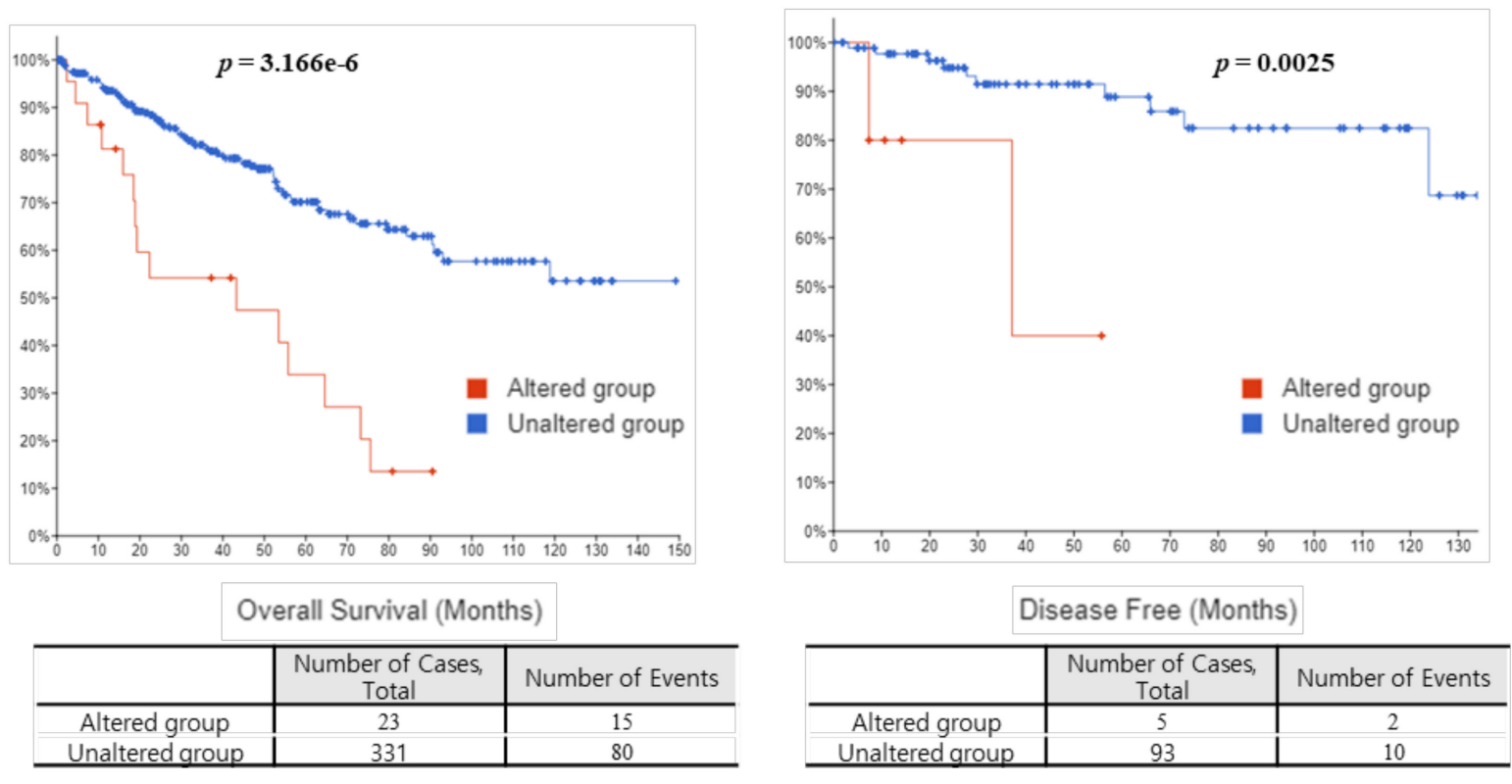

Figure 3. Survival analysis of the TCGA-KIRC data for all six survival genes (ADAMTS10, CARD6, NLRP2, OBSCN, SECISBP2L, and USP40). Overall survival (left) and disease-free survival (right) graphs based on data from (a) the Firehose Legacy and (b) the PanCancer Atlas.

The hazard ratios (HR) and $p$-values from the multivariate Cox regression analysis of six survival-specific genes and two clinical factors, $\mathrm{T}$ stage and metastasis, are shown in Table 7. Mutations in NLRP2 and OBSCN were significant in OS (HR $=5.72, p=0.01$ and $\mathrm{HR}=7.5, p=0.01$ ), whereas NLRP2 and USP40 genes were significant in DFS (HR $=9.62$ and $p=<0.005$ and $\mathrm{HR}=5.29, p=0.01$ ). Additionally, it was noticed that mutations in NLRP2 and OBSCN showed a statistically significant co-occurrence tendency ( $p=0.042$; Log2 Odds Ratio $>3$ ) from the Mutual Exclusivity analysis by cBioPortal (Zhiping Gu (2016), "cBioPortal," https:/ / ncihub.org/resources/1624, accessed on 15 November 2021). We identified that NLRP2 gene mutations are significant in both OS and DFS. In Figure 4, $N L R P 2$ and OBSCN gene mutations have a higher risk ratio in OS than the two clinical factors. Similarly, NLRP2 and USP40 mutations have a higher risk ratio in DFS than both clinical factors.

Table 7. Multivariate Cox regression analysis on survival in six survival-specific genes from TCGAKIRC (Firehose Legacy) data.

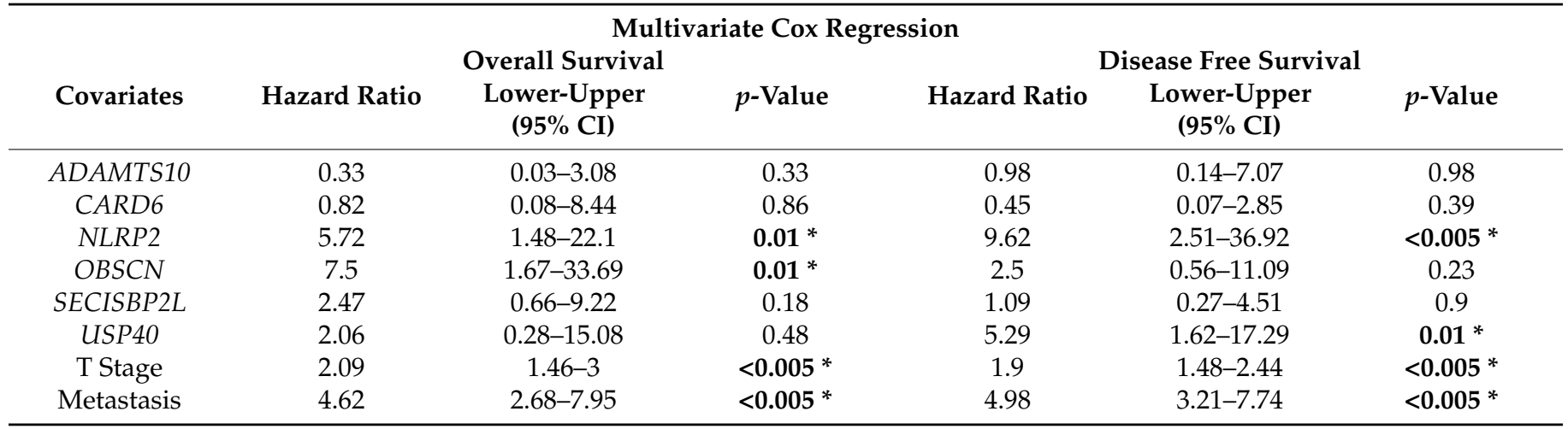

${ }^{*}$ is used of $p$-values $<0.05$. Statistical significant values are bolded. The $p$-values are from the Cox proportional hazard model. CI, confidence interval. 
(a)

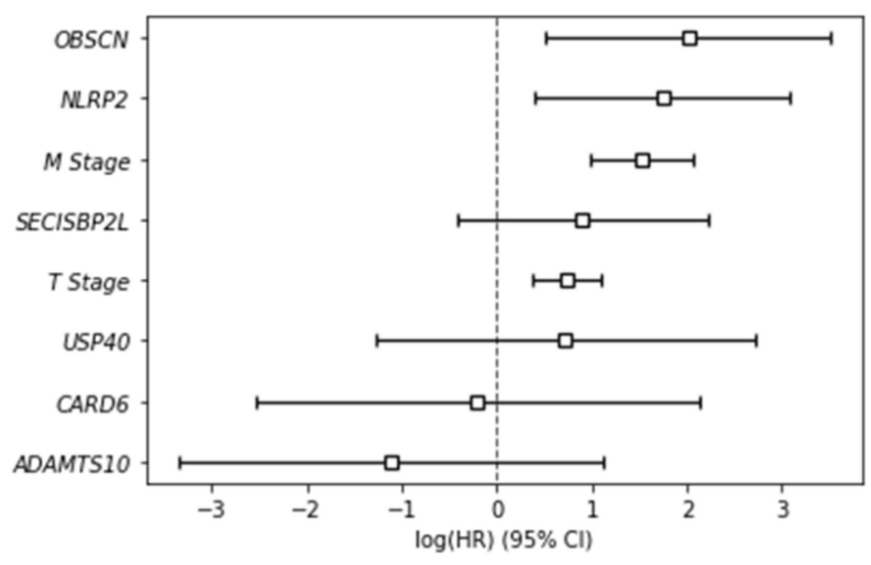

(b)

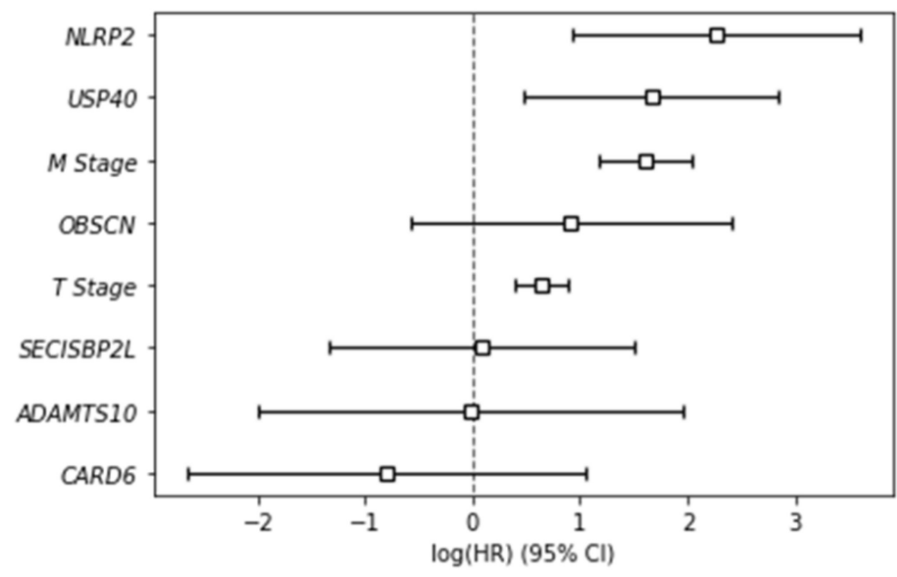

Figure 4. Multivariate forest plots on survival in TCGA_KIRC (Firehose Legacy) data by the multivariate Cox regression analysis: (a) overall survival and (b) disease-free survival. CI, confidence interval; $\log (\mathrm{HR})$, hazard ratio.

\section{Discussion}

Although various studies are being conducted on genes related to survival in RCC, there are few survival-specific genes that can be used as biomarkers in clinical practice. In this study, we identified mutations in 22 Korean ccRCC patients using an NGS gene panel, which includes a total of 123 survival-specific genes discovered through machine learning with TCGA-KIRC data. Moreover, the relationship between these mutated genes and clinicopathological findings was investigated. However, the results were different from our initial expectations. The gene panel used in this study was developed based on American data from TCGA, as there are still few studies of ccRCC based on Asian populations. Therefore, when this panel is tested in Korean or European cohorts, the different effects of genes on survival are thought to be due to genetic differences between races.

According to the TCGA report [7], the most frequent gene mutations in kidney cancer were VHL, PBRM1, SETD2, and BAP1. In this study of 22 Korean patients, the overall tendency of mutation frequencies was different from other reports. In particular, four genes, VHL (91\%), SETD2 (50\%), TSHZ3 (14\%), and SPEN (18\%), that are among the 16 top-ranked mutated genes were observed with higher frequencies in Koreans than in other populations (e.g., USA, Europe, and the rest of Asia). However, it is not appropriate to interpret the observed differences in mutation frequency between regions of these genes only as regional differences. Since sample sizes vary widely between regions, the possibility that sample sizes are also relevant should be considered. Since the number of Korean patients (22 patients) participating in this study was small, future cohort expansion is necessary to derive a more definitive interpretation.

The aim of this study was to determine the presence or absence of mutations in the 123 survival-specific genes in 22 Korean ccRCC patients and to determine whether these gene mutations were related to survival. From 11 genes of clinical importance, six (ADAMTS10, CARD6, NLRP2, OBSCN, SECISBP2L, and USP40) were derived from 123 survival-specific genes, and the remaining five (AKAP9, ARID1A, BAP1, KDM5C, and SETD2) were derived from the 16 top-ranked mutated genes in TCGA_KIRC. Our study showed that the mutations in the 11 genes were correlated with factors of OS, DFS, mortality, nuclear grade, sarcomatoid component, N-stage, sex, and tumor size.

In this NGS study of 22 Korean ccRCC patients, only the CARD 6 mutation was found to correlate with OS ( $p=0.04, r=-0.441)$ among 123 survival-specific genes. When we validated it with the publicly available data, there was a significant association between the CARD6 gene mutation and OS in both TCGA $(p=0.0003)$ and RECA-EU $(p=0.0005)$ dataset. Therefore, in all three datasets, CARD 6 was the only gene that showed a significant 
association with survival (OS) in Korean, TCGA-KIRC (American), and RECA-EU data (European).

However, to date, no association between CARD6 and survival has been reported in ccRCC or other cancers. CARD6 (Caspase recruitment domain family member 6) is a CARD-containing protein and plays pivotal roles in signal transduction, leading to apoptosis and NF-kB activation and inflammation [23]. CARD6 was reported to be involved in the activation of NF- $\kappa$ B signaling in gastrointestinal cancer [24]. In that study, neoexpression of CARD6 was related to activation of the NF-kB pathway and played a role in the development of esophageal, gastric, and colorectal tumors.

In addition to CARD6, we found 10 other genes (ADAMTS10, NLRP2, OBSCN, SECISBP2L, USP40, AKAP9, ARID1A, BAP1, KDM5C, and SETD2) that revealed correlation with OS and/or DFS. The BAP1 (BRCA1 associated protein-1) protein functions as a deubiquinating enzyme that regulates multiple cellular pathways related to tumorigenesis [25]. Our finding that mutant BAP1 was associated with DFS $(p=0.029, r=-0.465)$ was reported in other studies. In a comprehensive analysis of $445 \mathrm{ccRCC}$ cases from TCGA, BAP1 mutant ccRCC patients had worse OS $(p=0.035)$ and DFS $(p=0.036)$ than BAP1 wild-type patients [26]. BAP1 mutations were associated with worse cancer-specific survival (CSS) in both cohorts of the Memorial Sloan-Kettering Cancer Center $(p=0.002 ;$ HR 7.71; 95\% confidence interval (CI) 2.08-28.6) and TCGA ( $p=0.002$; HR 2.21; 95\% CI 1.35-3.63) [8,27]. Kapur et al. reported that tumors with negative $B A P 1$ expression (most were $B A P 1 \mathrm{mu}-$ tants) were related to shorter OS ( $p=0.001)$ [28], and $90 \%$ of BAP1-mutant tumors were related to a high (grades 3-4) Fuhrman grade [25]. In addition, as in the 145 patients with primary ccRCC from the University of Texas Southwestern Medical Center, patients with $B A P 1-m u t a t e d$ tumors had a significantly higher probability of death (HR 2.8, 1.4-5.9; $p=0.004)$ and were associated with a higher grade $(p=0.095)$, necrosis $(p=0.038)$, and advanced pathologic tumor stage and clinical stage ( $p=0.011$ and $p=0.003$, respectively), indicating that $B A P 1$-mutant tumors were associated with poor outcome [9]. In particular, a multivariate Cox proportional hazards model suggested that patients with NLRP2, USP40, and $O B S C N$ mutations have a higher mortality rate than other factors.

SECISBP2L (SECIS binding protein 2-like) is a paralogue of SECIS binding protein 2 and is known to play a role in selenoprotein expression [29]. The association of the SECISBP2L mutation found in our previous study using the TCGA_KIRC cohort with OS $(p=0.0005)$ and DFS $(p=0.0489)$ was not identified in 22 Korean patients. To date, there have been no reports of an association between SECISBP2L mutation and survival or mortality.

SETD2, a histone methyltransferase, plays an important role in the epigenetic control of gene expression [27]. SETD2 mutations, observed to be associated with nuclear grade $(p=0.035, r=0.451)$ in this study, were not reported in other studies of ccRCC. It was reported that mutations in SETD2 occurred in 3-12\% of ccRCC cases and were associated with poor clinical outcome [30]. Decreased SETD2 expression predicted unfavorable prognosis (larger tumor size and advanced pT stage) in patients with ccRCC [30]. SETD2 was associated with worse CSS in a TCGA cohort of 421 patients with primary ccRCC ( $p=0.036$; HR 1.68; 95\% CI 1.04-2.73) [8]. Hakimi et al. reported that tumors with mutations in $B A P 1, S E T D 2$, or KDM5C were more likely to present with stage III disease or higher $(p=0.001)$ [27]. We also found that SETD2 correlated with DFS in TCGA $(p=0.0201)$ and RECA-EU ( $p=0.0002)$. Similarly, a recent Chinese ccRCC study reported a correlation between a SETD2 mutation and a shorter DFS $(p=0.065)$ [6]. They performed deep sequencing, targeting 556 oncogenes in 105 tumor tissues to detect somatic mutations and clinicopathological effects in Chinese patients with ccRCC. Eight genes (BAP1, SETD2, $P T E N, E R B B 2, T P 53, C D K 8, T S C 1$, and SPEN) were associated with poor prognosis [27].

Our study showed a significant correlation of mutations in NLRP2, USP40, AKAP9 $(r=0.690, p=0.00038)$, and OBSCN $(r=0.474, p=0.026)$ with a sarcomatoid component. To our knowledge, there were no reports of associations between mutations in NLRP2, OBSCN, USP40, and AKAP9 genes and the sarcomatoid component. However, Liu et al. reported that the combined score according to SETD2/H3K36me3 expression was an independent 
prognostic factor for OS and DFS, which was associated with tumor size $(p=0.003), \mathrm{pT}$ stage $(p=0.043)$, and sarcomatoid component $(p=0.004)$ [30].

The nod-like receptor pyrin domain-containing proteins (NLRPS) are expressed by resident renal cells. Overexpression of the NLRP family member NLRP2 was found to contribute to the progression of renal failure by creating a vicious inflammatory cycle and decreasing the apoptotic cell rate [31]. Although we found that NLRP2 mutations are correlated with OS $(p=0.0029)$ and DFS $(p=3.09 \mathrm{e}-12)$ in the TCG-KIRC data, only a few studies have reported similar data, but not in RCC. A lung adenocarcinoma study has reported that low expression of NLRP2 is correlated with poor survival rates in lung adenocarcinoma ( $p=0.014)$ [32]. High expression of NLRP2 was associated with high risk in head and neck squamous cell carcinoma [33].

The OBSCN (Obscurin, cytoskeletal calmodulin and titin-interacting RhoGEF) gene encodes obscurin, which belongs to a family of giant cytoskeletal proteins [34]. OBSCN is regarded as a tumor suppressor for its ability to influence cellular integration and activate cancer initiation [35]. We discovered that OBSCN mutations were correlated with OS $(p=0.0096)$ and DFS ( $p=0.0048)$ in the TCGA-KIRC cohort, and with DFS $(p=3.28 \mathrm{e}-6)$ in the RECA-EU cohort, although there was no correlation of OBSCN mutations with survival in our 22 Korean ccRCC patients. Even though current studies on OBSCN mutations are minimal, the association between $O B S C N$ expression and clinical factors has been reported in other studies. A previous pRCC study reported that higher OBSCN expression was associated with poor survival outcomes $(p=0.021)$ [35]. A pancreatic adenosquamous carcinoma study reported that somatic mutations in OBSCN had a negative correlation with lymphatic metastasis in pancreatic adenosquamous carcinoma $(p=0.0339)$ [34]. Also, in our results, the OBSCN mutation was associated with female sex $(p=0.03, r=0.462)$, but similar results have not been reported.

The USP40 (Ubiquitin-specific peptidase 40) protein is a deubiquitinase, regulating the ubiquitination and degradation of CFLARL, which plays an important role in extrinsic ligand-induced apoptosis [36]. We found that the USP40 mutation was correlated with DFS ( $p=0.0003$ and $p=0.0269)$ in the TCGA_KIRC cohort (Firehose Legacy and PanCancer Atlas) and with OS ( $p=0.042)$ in the RECA-EU cohort. However, this was not validated in our 22 ccRCC patients.

The A-kinase anchor proteins ( $A K A P S)$ have the common function of binding to the regulatory subunit of protein kinase $\mathrm{A}$ and confining the holoenzyme to discrete locations within the cell. AKAP9 has been reported to be involved in the development or metastasis of several cancers [37]. We discovered that the $A K A P 9$ mutation was correlated with OS $(p=0.047)$ in the RECA-EU cohort, but not in the TCGA_KIRC cohort or our 22 Korean ccRCC patients.

In this study, we identified SECISBP2L ( $p=2.20 \mathrm{e}-16, r=1.000)$ and $\operatorname{KDM} 5 \mathrm{C}(p=0.00038$, $r=0.690)$ as genes associated with lymph node metastasis. A correlation between mutations of SECISBP2L or KDM5C and N-stage has not yet been reported in RCC. However, a recent colon cancer study stated that SECISBP2L showed high expression in the LN (+) group rather than the $\mathrm{LN}(-)$ group, and the expression level was significantly correlated with the survival rate [38]. KDM5C (Lysine demethylase 5C) is a JmjC domain-containing protein that removes methyl residues from methylated lysine 4 on histone H3 lysine 4. KDM5C has been proposed as an oncogene in many types of tumors [39]. We found that the KDM5C mutation was correlated with OS $(p=0.022)$ in a RECA-EU cohort, but not in a TCGAKIRC cohort or our 22 Korean ccRCC patients. In a ccRCC study, Hakimi et al. reported a significant connection $(p=0.001)$ between tumors with any of the three mutations of KDM5C, BAP1, or SETD2 and advanced stages [27]. It was reported that mutations in $K D M 5 C$ and BAP1 were significantly associated with renal vein invasion $(p=0.022$ and $p=0.046$, respectively) [40].

In our study, the two genes ADAMTS10 and ARID1A (both $p=0.004, r=0.585$ ) were associated with tumor size. The finding that ADAMTS10 mutations were associated with large tumor size has not been reported in other studies. However, there was a significant 
correlation between ARID1A mRNA expression and size $(p=0.03)$, grade $(p=0.03)$, and stage $(p=0.03)$ of tumor in a cCRCC study [41]. Liu et al. also reported that patients with low expression of SETD2 or H3K36me3 were prone to a large tumor size and advanced pT stage [30]. The A disintegrin and metalloproteinase with thrombospondin motif (ADAMTS) family of genes are a group of proteases found in both mammals and invertebrates [42] and reported to be involved in the occurrence and development of different cancers [43]. Although we discovered that ADAMTS10 mutations were correlated with OS $(p=0.0186)$ and DFS $(p=0.0004)$ in the TCGA-KIRC cohort, and with DFS $(p=0.0011)$ in the RECA-EU cohort, there was no correlation in the 22 Korean ccRCC patients. However, Liang et al. reported that the expression level of ADAMTS10 affects OS (HR $=1.45, p<0.026)$ and recurrence $(\mathrm{HR}=2.22, p=0.020)$ in patients with gastric cancer. The survival of patients with a high expression of ADAMTS10 was significantly lower than that of patients with a low expression [43].

The ARID1A (AT-rich interaction domain 1A), coding for the BAF250a subunit of BAF, is a tumor suppressor gene often mutated in RCC [41] and other types of carcinoma [44]. We found an association between $A R I D 1 A$ mutations and larger tumor sizes in this study $(p=0.004, r=0.585)$. According to a report on the association of ARID1A with tumor size in ccRCC [41], decreased $A R I D 1 A$ expression was associated with larger tumors $(\geq 7 \mathrm{~cm})$, high grades III and IV, and high stage III and IV (all: $p=0.03$ ). Additionally, we discovered that ARID1A mutation was correlated with OS $(p=0.0204)$ and DFS $(p=1.24 \mathrm{e}-5)$ in the RECA-EU cohort, but not in the TCGA-KIRC cohort or the 22 Korean cCRCC patients. A significantly lower expression of ARID1A than matched normal kidney cortex was reported in $67 \%$ of ccRCC (53 of 79), and a Kaplan-Meier analysis demonstrated significantly shorter OS among patients with $A R I D 1 A$-negative tumors $\left(x^{2}=9.14 ; p=0.003\right)$ [41]. The study of $A R I D 1 A$ in 290 cases of ccRCC in Korea showed that lower ARID1A expression was associated with higher nuclear grade $(p<0.001)$, higher pTNM stage $(p=0.013)$, and shorter CSS $(p=0.001)$ and progression-free survival $(p<0.001)$ [45].

Recently, radiogenomic studies of ccRCC showed associations between CT imaging features and mutations [40]. Mutations of $V H L$ were significantly associated with welldefined tumor margins $(p=0.013)$, nodular tumor enhancement $(p=0.021)$, and gross appearance of intratumoral vascularity $(p=0.018)$. The solid type of ccRCC differed in genotype significantly from the multicystic type. While mutations in BAP1, SETD2, and KDM5C were absent in the multicystic type, mutations of VHL $(p=0.016)$ and PBRM1 $(p=0.017)$ were significantly more common in the solid type [40].

This study confirmed that genetic mutations in particular genes are related to survival and clinicopathological findings in diverse forms. The NGS technique and populationbased analysis revealed that genetic differences, frequencies, and clinical differences in ccRCC exist between cohorts in East Asia, North America, and Europe. The limitation of the present study is that the panel was developed with genes derived from the TCGA-KIRC database due to a lack of studies on Asians with ccRCC. This accounts for the different results in survival-specific genes between the Korean and the Western cohorts. Another limitation is the size of the Korean samples used in this study. Further studies in large patient cohorts are needed to verify the clinicopathological significance of these novel mutations and to develop targeted therapeutics using these genetic markers. The authors expect that, based on the results of this study, a gene panel that predicts the prognosis of ccRCC patients will be developed and utilized by clinicians, including pathologists, urologists, oncologists, and radiation oncologists. However, many challenges remain to provide a reliable prognostic strategy for ccRCC patients.

In conclusion, we discovered and validated 11 survival-specific genes in ccRCC patients by using data from the TCGA-KIRC, RECA-EU, and Korean patients. To our knowledge, we are the first to find a correlation between CARD 6 mutations and OS in patients with ccRCC. The 11 genes, including CARD6, NLRP2, OBSCN, and USP40, might be useful diagnostic, prognostic, and therapeutic markers in ccRCC. 
Supplementary Materials: The following supporting information can be downloaded at: https: / / www.mdpi.com/article/10.3390/jpm12010113/s1, Table S1. (a): The performance of feature selection method_Information Gain (IG) depending on the weights and classifiers, Table S1. (b): The performance of feature selection method_Chi-squared depending on the sample sizes and classifiers, Table S1. (c): The performance of feature selection method_MRMR depending on the numbers of relevant genes and classifiers, Figure S1: Survival curves of top 100 genes by three feature selection methods (Information Gain, Chi-squared test, and MRMR), Table S2: A total of 123 survivalspecific mutations selected in TCGA-KIRC using three feature selection methods (Information Gain, Chi-squared test, and MRMR), Table S3: A total of 21 of top-ranked mutant genes above 5\% in TCGA-KIRC, Table S4: A total of 14 genes for other solid tumors, Table S5: The Pearson's correlation test revealed that the presence of a mutation in CARD6 was inversely related to the period of OS (Patient P10: 14 months, the shortest), Table S6: The Pearson's correlation test showed that the presence of the BAP1 mutation was moderately negatively related to the period of DFS (Patient P8: 28 months and alive, Patient P12: 0 month and alive, Patient P16: 1 month and dead, and Patient P22:1 month and dead), Table S7: The Pearson's correlation test revealed that the presence of a mutation in SECISBP2L was rigorously related to the spread of cancer cells to the lymph nodes and positively related to death (Patient P11: positive and dead), Table S8: The Pearson's correlation test revealed that the presence of a mutation in SETD2 was moderately related to nuclear grade (Patient P3: grade 4, Patient P5: grade 3, Patient P8: grade 3, Patient P11: grade 4, Patient P12: grade 4, Patient P18: grade 2, Patient P20: grade 3, Patient P21: grade 3 and Patient P22: grade 4), Table S9: The Pearson's correlation test revealed that the presence of a mutation in NLRP2 was strongly associated with sarcomatoid component (Patient P5: positive), Table S10: Although mutation in OBSCN and sex were moderately associated, we noticed that both patients had OBSCN mutation were female (Patient P6 and Patient P12) in this case. It is also observed that Patient P12 had sarcomatoid component (Patient P12: positive), Table S11: The Pearson's correlation test revealed that the presence of a mutation in USP40 was strongly associated with having sarcomatoid component (Patient P5: positive), Table S12: The Pearson's correlation test revealed that the presence of a mutation in AKAP9 was strongly associated with having sarcomatoid component (Patient P5: positive), Table S13: The Pearson's correlation test revealed that the presence of a mutation in KDM5C was strongly related to the fact that cancer tissue had spread to the nearby lymph nodes (P11: positive), Table S14: The Pearson's correlation test revealed that ADAMTS10 mutation was moderately associated with tumor size. Patient P18 had ADAMTS10 mutation showed the largest tumor size $(14.5 \mathrm{~cm})$ among 22 patients (size of tumors without mutation- mean: $6.2 \mathrm{~cm}$, median: $5.5 \mathrm{~cm}$ ), Table S15: The Pearson's correlation test revealed that $A R I D 1 A$ mutation is moderately associated with tumor size. Patient P18 had $A R I D 1 A$ mutation showed the largest tumor size $(14.5 \mathrm{~cm})$ among 22 patients.

Author Contributions: Conception and design, Y.C.; Development of methodology, Y.C., J.Y. and K.K.; Acquisition of data (provided animals, acquired and managed patients, provided facilities, etc.), Y.C., S.H., S.K., J.H. (Jinseon Han) and J.L.; Analysis and interpretation of data (e.g., statistical analysis, biostatistics, computational analysis), Y.C., J.H. (Jinseon Han), J.L., S.H., U.P., S.K.Y. and K.C.; Writing, review, and/or revision of the manuscript: Y.C., K.Y., Y.K., J.H. (Jia Hwang), H.K. and S.K.Y.; Administrative, technical, or material support (i.e., reporting or organizing data, constructing databases), Y.C., J.H. (Jia Hwang), H.K. and J.L.; Study supervision, Y.C. All authors have read and agreed to the published version of the manuscript.

Funding: This study was supported by the 2018 Research Fund of Seoul St. Mary's Hospital, The Catholic University of Korea (CUMC_SSMH_Research Fund_2018-03). The costs of publication of this article were defrayed in part by the payment of page charges. This article must therefore be hereby marked advertisement in accordance with 18 U.S.C. Section 1734 solely to indicate this fact. This work was supported by the Korea Medical Device Development Fund grant, funded by the Korea government (the Ministry of Science and ICT, the Ministry of Trade, Industry and Energy, the Ministry of Health \& Welfare, the Ministry of Food and Drug Safety) (Project Number: 9991006769, KMDF_PR_20200901_0096) (NTIS, KMDF_RnD_20200901_0096).

Institutional Review Board Statement: All procedures performed in this study were in accordance with the 1964 Helsinki declaration and its later amendments or comparable ethical standards, and with the ethical standards approved by the Institutional Review Board of Catholic University of Korea, Seoul St. Mary's Hospital (approval no. 2018-2550-0008, date of approval: 25 September 2020). 
Informed Consent Statement: Informed consent was obtained from all subjects involved in the study.

Data Availability Statement: This study was conducted with bio-resources from National Biobank of Korea, the Centers for Disease Control and Prevention, Republic of Korea (KBN-2019-019, approval date: 21 March 2019; Project Number, KC18SNSI0549).

Acknowledgments: We would like to thank the staff at the Machine Learning Research Laboratory in the Department of Computer Science and Engineering at Sogang University for their technical assistance on the machine learning with TCGA-KIRC data and the bioinformatics analysis. We are grateful to Insun Lee and Hyunjeung Choi, employees of NGeneBio Co., Ltd., for their help in the development and experiment of the ccRCC NGS gene panel.

Conflicts of Interest: Y.C. and S.H. are listed as co-inventors on patents for the NGS gene panel developed in this study, owned by The Catholic University of Korea. K.K. is a head of research institute of NGeneBio Co., Ltd. No commercial support was obtained in the planning or execution of the study. All authors declare no potential conflicts of interest.

Translational Relevance: Clear cell renal cell carcinoma (ccRCC) is known to have high tumor heterogeneity, probably due to variability in gene mutations. This study was to discover a genetic mutation related to the survival of cancer patients and to find biomarkers that can be used to predict the patient's prognosis and make treatment decisions in the clinic. Next-generation sequencing (NGS) is the most useful multigene assay for diagnosing mutations. Herein, we developed a customizedNGS gene panel for ccRCC using 156 genes, including 123 survival-specific genes newly discovered by machine learning and 21 top-ranked mutated genes above $5 \%$ in The Cancer Genome AtlasKidney Renal Clear Cell Carcinoma (TCGA-KIRC). The 11 survival-specific genes, including CARD6, discovered and verified here were based on data from TCGA-KIRC, Renal Cell Cancer-European Union (RECA-EU) and Korean patients, which could be used to provide precision medicine and personalized treatment to patients with ccRCC.

\author{
Abbreviations \\ ccRCC clear cell renal cell carcinoma \\ TCGA The Cancer Genome Atlas \\ ngs next-generation sequencing \\ RECA-EU Renal Cell Cancer-European Union
}

\title{
References
}

1. Shuch, B.; Amin, A.; Armstrong, A.J.; Eble, J.N.; Ficarra, V.; López-Beltrán, A.; Martignoni, G.; Rini, B.I.; Kutikov, A. Understanding Pathologic Variants of Renal Cell Carcinoma: Distilling Therapeutic Opportunities from Biologic Complexity. Eur. Urol. 2015, 67, 85-97. [CrossRef]

2. $\quad$ Byun, S.S.; Hong, S.K.; Lee, S.; Kook, H.R.; Lee, E.; Kim, H.H.; Kwak, C.; Ku, J.H.; Jeong, C.W.; Lee, J.Y.; et al. The establishment of KORCC (KOrean Renal Cell Carcinoma) database. Investig. Clin. Urol. 2016, 57, 50-57. [CrossRef] [PubMed]

3. Suh, J.; Jeong, C.W.; Choi, S.; Ku, J.H.; Kim, H.H.; Kim, K.; Kwak, C. Sharing the initial experience of pan-cancer panel analysis in high-risk renal cell carcinoma in the Korean population. BMC Urol. 2020, 20, 125. [CrossRef]

4. Beksac, A.T.; Paulucci, D.J.; Blum, K.A.; Yadav, S.S.; Sfakianos, J.P.; Badani, K.K. Heterogeneity in renal cell carcinoma. Urol. Oncol. Semin. Orig. Investig. 2017, 35, 507-515. [CrossRef] [PubMed]

5. Kan, Z.; Jaiswal, B.S.; Stinson, J.; Janakiraman, V.; Bhatt, D.; Stern, H.M.; Yue, P.; Haverty, P.M.; Bourgon, R.; Zheng, J.; et al. Diverse somatic mutation patterns and pathway alterations in human cancers. Nature 2010, 466, 869-873. [CrossRef] [PubMed]

6. Bi, H.; Yin, J.P.; Zhou, L.; Wu, Y.Q.; Ge, L.Y.; Lu, M.; Liu, L.; Zhang, H.; Zhao, Y.; Liu, C.; et al. Clinicopathological and prognostic impact of somatic mutations in Chinese patients with clear cell renal cell carcinoma. Transl. Androl. Urol. 2020, 9, $2751-2763$. [CrossRef] [PubMed]

7. Cancer Genome Atlas Research Network. Comprehensive molecular characterization of clear cell renal cell carcinoma. Nature 2013, 499, 43-49. [CrossRef]

8. Hakimi, A.A.; Ostrovnaya, I.; Reva, B.; Schultz, N.; Chen, Y.-B.; Gonen, M.; Liu, H.; Takeda, S.; Voss, M.H.; Tickoo, S.K.; et al. Adverse Outcomes in Clear Cell Renal Cell Carcinoma with Mutations of 3p21 Epigenetic Regulators BAP1 and SETD2: A Report by MSKCC and the KIRC TCGA Research Network. Clin. Cancer Res. 2013, 19, 3259-3267. [CrossRef]

9. Kapur, P.; Peña-Llopis, S.; Christie, A.; Zhrebker, L.; Pavía-Jiménez, A.; Rathmell, W.K.; Xie, X.-J.; Brugarolas, J. Effects on survival of BAP1 and PBRM1 mutations in sporadic clear-cell renal-cell carcinoma: A retrospective analysis with independent validation. Lancet Oncol. 2013, 14, 159-167. [CrossRef] 
10. Lin, P.-H.; Chuang, C.-K.; Yu, K.-J.; Kan, H.-C.; Liu, C.-Y.; Lu, Y.-C.; Chang, Y.-H.; Shao, I.-H.; Pang, S.-T. Genomic characterization of clear cell renal cell carcinoma using targeted gene sequencing. Oncol. Lett. 2021, 21, 169. [CrossRef]

11. Dalgliesh, G.L.; Furge, K.; Greenman, C.; Chen, L.; Bignell, G.; Butler, A.; Davies, H.; Edkins, S.; Hardy, C.; Latimer, C.; et al. Systematic sequencing of renal carcinoma reveals inactivation of histone modifying genes. Nature 2010, 463, 360-363. [CrossRef]

12. Boycott, K.M.; Vanstone, M.R.; Bulman, D.E.; MacKenzie, A.E. Rare-disease genetics in the era of next-generation sequencing: Discovery to translation. Nat. Rev. Genet. 2013, 14, 681-691. [CrossRef]

13. Legati, A.; Reyes, A.; Nasca, A.; Invernizzi, F.; Lamantea, E.; Tiranti, V.; Garavaglia, B.; Lamperti, C.; Ardissone, A.; Moroni, I.; et al. New genes and pathomechanisms in mitochondrial disorders unraveled by NGS technologies. Biochim. Biophys. Acta 2016, 1857, 1326-1335. [CrossRef]

14. DePristo, M.A.; Banks, E.; Poplin, R.; Garimella, K.V.; Maguire, J.R.; Hartl, C.; Philippakis, A.A.; Del Angel, G.; Rivas, M.A.; Hanna, M.; et al. A framework for variation discovery and genotyping using next-generation DNA sequencing data. Nat. Genet. 2011, 43, 491-498. [CrossRef]

15. Poplin, R.; Chang, P.-C.; Alexander, D.; Schwartz, S.; Colthurst, T.; Ku, A.; Newburger, D.; Dijamco, J.; Nguyen, N.; Afshar, P.T.; et al. A universal SNP and small-indel variant caller using deep neural networks. Nat. Biotechnol. 2018, 36, 983-987. [CrossRef]

16. Cingolani, P.; Platts, A.; Wang, L.L.; Coon, M.; Nguyen, T.; Wang, L.; Land, S.J.; Lu, X.; Ruden, D.M. A program for annotating and predicting the effects of single nucleotide polymorphisms, SnpEff: SNPs in the genome of Drosophila melanogaster strain w(1118); iso-2; iso-3. Fly 2012, 6, 80-92. [CrossRef] [PubMed]

17. Karczewski, K.J.; Francioli, L.C.; Tiao, G.; Cummings, B.B.; Alfoldi, J.; Wang, Q.; Collins, R.L.; Laricchia, K.M.; Ganna, A.; Birnbaum, D.P.; et al. The mutational constraint spectrum quantified from variation in 141,456 humans. Nature 2020,581, $434-443$. [CrossRef]

18. Consortium, G.P.; Auton, A.; Brooks, L.D.; Durbin, R.M.; Garrison, E.P.; Kang, H.M. A global reference for human genetic variation. Nature 2015, 526, 68-74. [CrossRef] [PubMed]

19. Tate, J.G.; Bamford, S.; Jubb, H.C.; Sondka, Z.; Beare, D.M.; Bindal, N.; Boutselakis, H.; Cole, C.G.; Creatore, C.; Dawson, E.; et al. COSMIC: The Catalogue of Somatic Mutations In Cancer. Nucleic Acids Res. 2019, 47, D941-D947. [CrossRef] [PubMed]

20. Landrum, M.J.; Lee, J.M.; Riley, G.R.; Jang, W.; Rubinstein, W.S.; Church, D.M.; Maglott, D.R. ClinVar: Public archive of relationships among sequence variation and human phenotype. Nucleic Acids Res. 2014, 42, D980-D985. [CrossRef]

21. Kim, Y.; Han, B.G.; KoGES Group. Cohort Profile: The Korean Genome and Epidemiology Study (KoGES) Consortium. Int. J. Epidemiol. 2017, 46, e20. [CrossRef] [PubMed]

22. Moon, S.; Kim, Y.J.; Han, S.; Hwang, M.Y.; Shin, D.M.; Park, M.Y.; Lu, Y.; Yoon, K.; Jang, H.-M.; Kim, Y.K.; et al. The Korea Biobank Array: Design and Identification of Coding Variants Associated with Blood Biochemical Traits. Sci. Rep. 2019, 9, 1382. [CrossRef]

23. Dufner, A.; Pownall, S.; Mak, T.W. Caspase recruitment domain protein 6 is a microtubule-interacting protein that positively modulates NF-kB activation. Proc. Natl. Acad. Sci. USA 2006, 103, 988-993. [CrossRef] [PubMed]

24. Kim, S.S.; Ahn, C.H.; Kang, M.R.; Kim, Y.R.; Kim, H.S.; Yoo, N.J.; Lee, S.H. Expression of CARD6, an NF-kappaB activator, in gastric, colorectal and oesophageal cancers. Pathology 2010, 42, 50-53. [CrossRef] [PubMed]

25. Joseph, R.W.; Kapur, P.; Bs, D.J.S.; Eckel-Passow, J.E.; Parasramka, M.J.; Ho, T.; Cheville, J.C.; Frenkel, E.; Rakheja, D.; Brugarolas, J.; et al. Loss of BAP1 protein expression is an independent marker of poor prognosis in patients with low-risk clear cell renal cell carcinoma. Cancer Am. Cancer Soc. 2014, 120, 1059-1067. [CrossRef]

26. Jin, S.M.; Wu, J.L.; Zhu, Y.; Gu, W.J.; Wan, F.N.; Xiao, W.; Dai, B.; Zhang, H.; Shi, G.; Shen, Y.; et al. Comprehensive Analysis of BAP1 Somatic Mutation in Clear Cell Renal Cell Carcinoma to Explore Potential Mechanisms in Silico. J. Cancer 2018, 9, 4108-4116. [CrossRef]

27. Hakimi, A.A.; Chen, Y.B.; Wren, J.; Gonen, M.; Abdel-Wahab, O.; Heguy, A.; Liu, H.; Takeda, S.; Tickoo, S.K.; Reuter, V.E.; et al. Clinical and Pathologic Impact of Select Chromatin-modulating Tumor Suppressors in Clear Cell Renal Cell Carcinoma. Eur. Urol. 2013, 63, 848-854. [CrossRef]

28. Kapur, P.; Christie, A.; Raman, J.D.; Then, M.T.; Nuhn, P.; Buchner, A.; Bastian, P.; Seitz, C.; Shariat, S.F.; Bensalah, K.; et al. BAP1 Immunohistochemistry Predicts Outcomes in a Multi-Institutional Cohort with Clear Cell Renal Cell Carcinoma. J. Urol. 2014, 191, 603-610. [CrossRef]

29. Donovan, J.; Copeland, P.R. Evolutionary history of selenocysteine incorporation from the perspective of SECIS binding proteins. BMC Evol. Biol. 2009, 9, 229. [CrossRef]

30. Liu, W.S.; Fu, Q.; An, H.M.; Chang, Y.; Zhang, W.J.; Zhu, Y.; Xu, L.; Xu, J. Decreased Expression of SETD2 Predicts Unfavorable Prognosis in Patients With Nonmetastatic Clear-Cell Renal Cell Carcinoma. Medicine 2015, 94, e2004. [CrossRef]

31. Rossi, M.N.; Pascarella, A.; Licursi, V.; Caiello, I.; Taranta, A.; Rega, L.R.; Levtchenko, E.; Emma, F.; De Benedetti, F.; Prencipe, G. NLRP2 Regulates Proinflammatory and Antiapoptotic Responses in Proximal Tubular Epithelial Cells. Front. Cell Dev. Biol. 2019, 7, 252. [CrossRef] [PubMed]

32. Lin, W.; Chen, Y.; Wu, B.; Li, Z. Identification of the pyroptosisrelated prognostic gene signature and the associated regulation axis in lung adenocarcinoma. Cell Death Discov. 2021, 7, 161. [CrossRef]

33. Wang, J.; Chen, X.; Tian, Y.; Zhu, G.; Qin, Y.; Pi, L.; Wei, M.; Liu, G.; Li, Z.; Chen, C.; et al. Six-gene signature for predicting survival in patients with head and neck squamous cell carcinoma. Aging 2020, 12,767-783. [CrossRef]

34. Ma, H.Y.; Song, B.; Guo, S.W.; Li, G.; Jin, G. Identification of germline and somatic mutations in pancreatic adenosquamous carcinoma using whole exome sequencing. Cancer Biomark. 2020, 27, 389-397. [CrossRef] 
35. Zhang, C.J.; Zheng, Y.X.; Li, X.; Hu, X.; Qi, F.; Luo, J. Genome-wide mutation profiling and related risk signature for prognosis of papillary renal cell carcinoma. Ann. Transl. Med. 2019, 7, 427. [CrossRef] [PubMed]

36. An, W.; Yao, S.; Sun, X.; Hou, Z.; Lin, Y.; Su, L.; Liu, X. Glucocorticoid modulatory element-binding protein 1 (GMEB1) interacts with the deubiquitinase USP40 to stabilize CFLAR(L) and inhibit apoptosis in human non-small cell lung cancer cells. J. Exp. Clin. Cancer Res. 2019, 38, 181. [CrossRef]

37. Hu, Z.-Y.; Liu, Y.-P.; Xie, L.-Y.; Wang, X.-Y.; Yang, F.; Chen, S.-Y.; Li, Z.-G. AKAP-9 promotes colorectal cancer development by regulating Cdc42 interacting protein 4. Biochim. Biophys. Acta BBA Mol. Basis Dis. 2016, 1862, 1172-1181. [CrossRef] [PubMed]

38. Han, S.W.; Ahn, J.Y.; Lee, S.; Noh, Y.S.; Jung, H.C.; Lee, M.H.; Park, H.J.; Chun, H.J.; Choi, S.J.; Kim, E.S.; et al. Gene expression network analysis of lymph node involvement in colon cancer identifies AHSA2, CDK10, and CWC22 as possible prognostic markers. Sci. Rep.-UK 2020, 10, 7170. [CrossRef]

39. Ji, X.N.; Jin, S.; Qu, X.T.; Li, K.J.; Wang, H.J.; He, H.; Guo, F.; Dong, L. Lysine-specific demethylase 5C promotes hepatocellular carcinoma cell invasion through inhibition BMP7 expression. BMC Cancer 2015, 15, 801. [CrossRef]

40. Karlo, C.A.; Di Paolo, P.L.; Chaim, J.; Hakimi, A.A.; Ostrovnaya, I.; Russo, P.; Hricak, H.; Motzer, R.; Hsieh, J.J.; Akin, O.; et al. Radiogenomics of Clear Cell Renal Cell Carcinoma: Associations between CT Imaging Features and Mutations. Radiology 2014, 270, 464-471. [CrossRef]

41. Lichner, Z.; Scorilas, A.; White, N.M.A.; Girgis, A.H.; Rotstein, L.; Wiegand, K.C.; Latif, A.; Chow, C.; Huntsman, D.; Yousef, G.M. The Chromatin Remodeling Gene ARID1A Is a New Prognostic Marker in Clear Cell Renal Cell Carcinoma. Am. J. Pathol. 2013, 182, 1163-1170. [CrossRef]

42. Porter, S.; Clark, I.M.; Kevorkian, L.; Edwards, D.R. The ADAMTS metalloproteinases. Biochem. J. 2005, 386, 15-27. [CrossRef]

43. Liang, L.; Zhu, J.-H.; Chen, G.; Qin, X.-G.; Chen, J.-Q. Prognostic Values for the mRNA Expression of the ADAMTS Family of Genes in Gastric Cancer. J. Oncol. 2020, 2020, 9431560. [CrossRef] [PubMed]

44. Li, L.; Li, M.; Jiang, Z.; Wang, X. ARID1A Mutations Are Associated with Increased Immune Activity in Gastrointestinal Cancer. Cells 2019, 8, 678. [CrossRef] [PubMed]

45. Park, J.H.; Lee, C.; Suh, J.H.; Chae, J.Y.; Kim, H.W.; Moon, K.C. Decreased ARID1A expression correlates with poor prognosis of clear cell renal cell carcinoma. Hum. Pathol. 2015, 46, 454-460. [CrossRef] [PubMed] 